Mathematical Modelling and Analysis

Volume 20 Number 1, January 2015, 53-75

http://dx.doi.org/10.3846/13926292.2015.1000988

(c) Vilnius Gediminas Technical University, 2015
Publisher: Taylor\&Francis and VGTU

http://www.tandfonline.com/TMMA

ISSN: $1392-6292$

eISSN: $1648-3510$

\title{
Approximate Transmission Conditions for a Poisson Problem at Mid-Diffusion
}

\section{Khaled El-Ghaouti Boutarene}

\author{
USTHB, Faculty of Mathematics, AMNEDP Laboratory \\ PO Box 32, El Alia 16111, Bab Ezzouar, Algiers, Algeria \\ E-mail: kboutarene@usthb.dz \\ E-mail: boutarenekhaled@yahoo.fr
}

Received November 29, 2013; revised December 4, 2014; published online January 15, 2015

\begin{abstract}
This work consists in the asymptotic analysis of the solution of Poisson equation in a bounded domain of $\mathbb{R}^{P}(P=2,3)$ with a thin layer. We use a method based on hierarchical variational equations to derive an explicitly asymptotic expansion of the solution with respect to the thickness of the thin layer. We determine the first two terms of the expansion and prove the error estimate made by truncating the expansion after a finite number of terms. Next, using the first two terms of the asymptotic expansion, we show that we can model the effect of the thin layer by a problem with transmission conditions of order two.
\end{abstract}

Keywords: asymptotic analysis, asymptotic expansion, approximate transmission conditions, thin layer, Poisson equation.

AMS Subject Classification: 35C20; 35B40; 78M35; 41A60; 35J25; 78M30.

\section{Introduction}

This paper deals with the study of the asymptotic behavior of the solution of the Poisson equation in a bounded domain $\Omega$ of $\mathbb{R}^{P}(P=2,3)$ consisting of two sub-domains separated by a thin layer of thickness $\delta$ (destined to tend to 0 ). The mesh of these thin geometries leads to systems with a condition number that increases significantly (cf. [27]). To overcome this difficulty, we adopt asymptotic methods to model the effect of the thin layer by problems with appropriate transmission conditions. These methods are widely used in different frameworks (cf., e.g., $[1,2,7,9,10,12,17,18]$ ). Especially, effective boundary conditions have been obtained for the Helmholtz and Maxwell equations in $[3,4,15,16]$ in smooth geometries and in [11] for Poisson problem in a polygonal domain. The case of approximate transmission conditions has been studied in $[6,13,21,25,26]$ for thin layer and in $[13,22,23]$ for rough and periodic thin layer. In [24, Part II] Schmidt derived an approximation of the solution inside the thin layer based on families of functions called optimal basis functions. 
Our motivation comes from [20,25], in which the authors have worked on problems of electromagnetic and biological origins. Our Problem is inspired from the thesis of Poignard [20, Chapter 2]. He considered a cell immersed in an ambient medium and studied the electric field in the transverse magnetic (TM) mode at mid-frequency.

Let us give now precise notations. The domain $\Omega$ is composed of smooth sub-domains: an open bounded subset $\Omega_{\text {int, } \delta}$ with regular boundary $\Gamma_{\delta, 1}$, an exterior domain $\Omega_{\text {ext, } \delta}$ with disjoint regular boundaries $\Gamma_{\delta, 2}$ and $\partial \Omega$, and a membrane $\Omega_{\delta}$ (thin layer) of thickness $\delta$ separating $\Omega_{\text {int, } \delta}$ from $\Omega_{\text {ext }, \delta}$ (see Fig. 1).

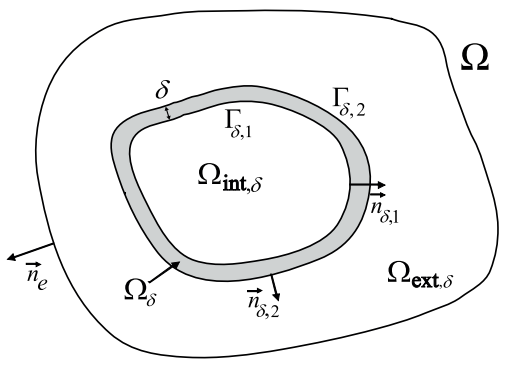

Figure 1. Geometric data.

Define the piecewise regular function $\alpha$ by

$$
\alpha(x)= \begin{cases}\alpha_{e} & \text { if } x \in \Omega_{\mathrm{ext}, \delta} \\ \alpha_{\delta} & \text { if } x \in \Omega_{\delta} \\ \alpha_{i} & \text { if } x \in \Omega_{\mathrm{int}, \delta}\end{cases}
$$

where $\alpha_{e}, \alpha_{\delta}$ and $\alpha_{i}$ are strictly positive constants satisfying $\alpha_{i}<\alpha_{\delta}<\alpha_{e}$ or $\alpha_{e}<\alpha_{\delta}<\alpha_{i}$ which correspond to the case of mid-diffusion. For a given $f$ in $\mathcal{C}^{\infty}(\bar{\Omega})$, we are interested in the solution $u_{\delta}$ of the following diffusion problem

$$
\begin{cases}-\operatorname{div}\left(\alpha \nabla u_{\delta}\right)=f & \text { in } \Omega, \\ u_{\delta \mid \partial \Omega}=0 & \text { on } \partial \Omega .\end{cases}
$$

The main result of this paper consists of an asymptotic expansion of the solution $u_{\delta}$ of Problem (1.1) in presence of a thin layer in terms of its thickness $\delta$ and the derivation of the associated approximated model of order 1 . The position of the limit interface $\Gamma$ (see Fig. 2 and Fig. 3) for $\delta$ tends to 0 is varied and a particular choice of $\Gamma$ leads to a simple problem involving the Poisson equation with transmission conditions of order two on the interface $\Gamma$ which lies between $\Gamma_{\delta, 1}$ and $\Gamma_{\delta, 2}$.

We use an approach based on variational equations (cf. $[3,6]$ ) to derive transmission conditions with tangential derivatives of order two on $\Gamma$, modeling the effect of the thin layer. However, it seems that the existence and uniqueness of the solution of this problem is not obvious. Therefore, we rewrite the problem as a pseudodifferential equation (cf. [5]) and show that in the case of mid-diffusion, we can find the appropriate position of the surface $\Gamma$ 


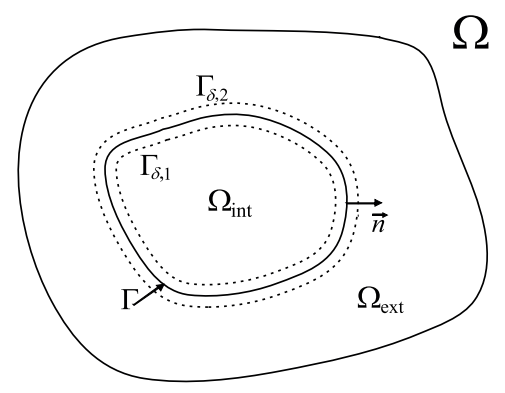

Figure 2. Geometry of the studied problem.

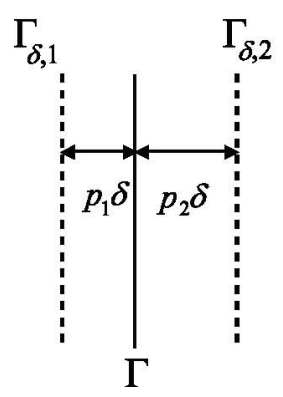

Figure 3. The thin layer $\Omega_{\delta}$.

to solve this equation. The problems are similar in $2 \mathrm{D}$ and $3 \mathrm{D}$. We treat the three-dimensional case and we give a remark to the two-dimensional case.

The present paper is organized as follows. In Section 2, we give the statement of the model problem considered. In Section 3, we collect basic results of differential geometry of surfaces. Sections 4 and 5 are devoted to the asymptotic analysis of our problem. We present, in Section 4, hierarchical variational equations suited to the construction of a formal asymptotic expansion up to any order, while Section 5 focuses on the convergence of this ansatz. With the help of the asymptotic expansion of the solution $u_{\delta}$, we model, in the last section, the effect of the thin layer by a problem with appropriate transmission conditions.

\section{Problem Setting}

We consider a parallel surface $\Gamma$ to $\Gamma_{\delta, 1}$ and $\Gamma_{\delta, 2}$ dividing $\Omega_{\delta}$ into two thin layers $\Omega_{\delta, 1}$ and $\Omega_{\delta, 2}$ of thickness respectively $p_{1} \delta$ and $p_{2} \delta$, where $p_{1}$ and $p_{2}$ are nonnegative real numbers satisfying $p_{1}+p_{2}=1$ and such that $p_{1}$ and $p_{2}$ belong to a small neighborhood of $1 / 2$ (see Fig. 2 and Fig. 3 ). The term small neighborhood means that the constants $p_{1}$ and $p_{2}$ are not too close to 1 or 0 , in order to avoid having a layer too thin compared to the other because the following analysis does not lend itself to this case. Let us denote by $u_{\text {ext }, \delta}$, $u_{d_{2}, \delta}, u_{d_{1}, \delta}$ and $u_{\text {int }, \delta}$ the restrictions of $u_{\delta}$ respectively to the domains $\Omega_{\text {ext }, \delta}$, $\Omega_{\delta, 2}, \Omega_{\delta, 1}$ and $\Omega_{\mathrm{int}, \delta}$. Under the aforementioned assumptions, we investigate in $H^{1}(\Omega)$ the solution $u_{\delta}$ of the following problem

$$
\begin{cases}-\operatorname{div}\left(\alpha_{e} \nabla u_{\mathrm{ext}, \delta}\right)=f_{\mid \Omega_{\mathrm{ext}, \delta}} & \text { in } \Omega_{\mathrm{ext}, \delta}, \\ -\operatorname{div}\left(\alpha_{\delta} \nabla u_{d_{2}, \delta}\right)=f_{\mid \Omega_{\delta, 2}} & \text { in } \Omega_{\delta, 2}, \\ -\operatorname{div}\left(\alpha_{\delta} \nabla u_{d_{1}, \delta}\right)=f_{\mid \Omega_{\delta, 1}} & \text { in } \Omega_{\delta, 1}, \\ -\operatorname{div}\left(\alpha_{i} \nabla u_{\mathrm{int}, \delta}\right)=f_{\mid \Omega_{\mathrm{int}, \delta}} & \text { in } \Omega_{\mathrm{int}, \delta}, \\ u_{\mathrm{ext}, \delta \mid \partial \Omega}=0 & \text { on } \partial \Omega\end{cases}
$$

with transmission conditions

$$
\begin{cases}u_{d_{2}, \delta \mid \Gamma_{\delta, 2}}=u_{\mathrm{ext}, \delta \mid \Gamma_{\delta, 2}} & \text { on } \Gamma_{\delta, 2} \\ \alpha_{\delta} \partial_{\mathbf{n}_{\delta, 2}} u_{d_{2}, \delta \mid \Gamma_{\delta, 2}}=\alpha_{e} \partial_{\mathbf{n}_{\delta, 2}} u_{\mathrm{ext}, \delta \mid \Gamma_{\delta, 2}} & \text { on } \Gamma_{\delta, 2}\end{cases}
$$




$$
\begin{cases}u_{d_{1}, \delta \mid \Gamma}=u_{d_{2}, \delta \mid \Gamma} & \text { on } \Gamma, \\ \alpha_{\delta} \partial_{\mathbf{n}} u_{d_{1}, \delta \mid \Gamma}=\alpha_{\delta} \partial_{\mathbf{n}} u_{d_{2}, \delta \mid \Gamma} & \text { on } \Gamma, \\ u_{\text {int }, \delta \mid \Gamma_{\delta, 1}}=u_{d_{1}, \delta \mid \Gamma_{\delta, 1}} & \text { on } \Gamma_{\delta, 1}, \\ \alpha_{i} \partial_{\mathbf{n}_{\delta, 1}} u_{\text {int }, \delta \mid \Gamma_{\delta, 1}}=\alpha_{\delta} \partial_{\mathbf{n}_{\delta, 1}} u_{d_{1}, \delta \mid \Gamma_{\delta, 1}} & \text { on } \Gamma_{\delta, 1},\end{cases}
$$

where $\partial_{\mathbf{n}_{\delta, 1}}, \partial_{\mathbf{n}}, \partial_{\mathbf{n}_{\delta, 2}}$ and $\partial_{\mathbf{n}_{e}}$ denote the derivatives in the direction of the unit normal vectors $\mathbf{n}, \mathbf{n}_{\delta, 1}, \mathbf{n}_{\delta, 2}$ and $\mathbf{n}_{e}$ to $\Gamma_{\delta, 1}, \Gamma, \Gamma_{\delta, 2}$ and $\partial \Omega$ respectively (see Fig. 2). The following theorem gives the existence and uniqueness of the solution $u_{\delta}$ to Problem (2.1).

Theorem 1. Problem (2.1) admits a unique solution $u_{\delta}$ in $H^{1}(\Omega)$. Furthermore, there exists a constant $c$ independent of $\delta$ such that

$$
\left\|u_{\delta}\right\|_{H^{1}(\Omega)} \leq c\|f\|_{L^{2}(\Omega)} .
$$

Proof. Existence and uniqueness of $u_{\delta}$ follow from Lax-Milgram lemma [8].

\section{Recalls of Differential Geometry}

The goal of this section is to define and to collect the main features of differential geometry [14] (see also [19]) in order to formulate our problem in a fixed domain (independent of $\delta$ ) which is a key tool to determine the asymptotic expansion of the solution $u_{\delta}$. In the sequel, the greek indice $\beta$ takes the values 1 and 2 . Let $I_{\delta, 1}=(-\delta, 0)$ and $I_{\delta, 2}=(0, \delta)$. We parameterize the thin shell $\Omega_{\delta, \beta}$ by the manifold $\Gamma \times I_{\delta, \beta}$ through the mapping $\psi_{\beta}$ defined by

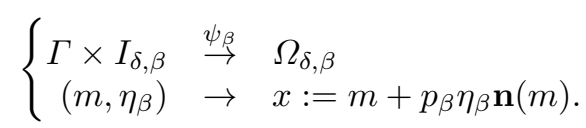

As well-known [14], if the thickness of $\Omega_{\delta, \beta}$ is small enough, $\psi_{\beta}$ is a $C^{\infty}$ diffeomorphism of manifolds and it is also known [21, Remark 2.1] that the normal vector $\mathbf{n}_{\delta, \beta}$ to $\Gamma_{\delta, \beta}$ can be identified to $\mathbf{n}$. To each function $v_{\beta}$ defined on $\Omega_{\delta, \beta}$, we associate the function $\widetilde{v}_{\beta}$ defined on $\Gamma \times I_{\delta, \beta}$ by

$$
\left\{\begin{aligned}
\widetilde{v}_{\beta}\left(m, \eta_{\beta}\right) & :=v_{\beta}(x), \\
x & =\psi_{\beta}\left(m, \eta_{\beta}\right),
\end{aligned}\right.
$$

then, we have

$$
\nabla v_{\beta}=\left(I+p_{\beta} \eta_{\beta} \mathcal{R}\right)^{-1} \nabla_{\Gamma} \widetilde{v}_{\beta}+p_{\beta}^{-1} \frac{\partial \widetilde{v}_{\beta}}{\partial \eta_{\beta}} \mathbf{n},
$$

where $\nabla_{\Gamma} \widetilde{v}_{\beta}(m)$ and $\mathcal{R}$ are respectively the surface gradient of $\widetilde{v}$ at $m \in \Gamma$ and the curvature operator $\mathcal{R}$ of $\Gamma$ at point $m$. The volume element on the thin shell $\Omega_{\delta, \beta}$ is given by

$$
d \Omega_{\delta, \beta}=p_{\beta} \operatorname{det}\left(I+p_{\beta} \eta_{\beta} \mathcal{R}\right) d \Gamma d \eta_{\beta} .
$$


Now, we introduce the scaling $s_{\beta}=\eta_{\beta} / \delta$, and the intervals $I_{1}=(-1,0)$ and $I_{2}=(0,1)$ such that the $C^{\infty}$-diffeomorphism $\Phi_{\beta}$, defined by

$$
\left\{\begin{array}{rll}
\Omega^{\beta}:=\Gamma \times I_{\beta} & \stackrel{\Phi_{\beta}}{\rightarrow} \Omega_{\delta, \beta} \\
\left(m, s_{\beta}\right) & \rightarrow & x:=m+\delta p_{\beta} s_{\beta} \mathbf{n}(m)
\end{array}\right.
$$

parameterizes the thin shell $\Omega_{\delta, \beta}$. To any function $v_{\beta}$ defined on $\Omega_{\delta, \beta}$, we associate the function $v^{[\beta]}$ defined on $\Omega^{\beta}$ through

$$
\left\{\begin{aligned}
v^{[\beta]}\left(m, s_{\beta}\right) & :=v_{\beta}(x), \\
x & =\Phi_{\beta}\left(m, s_{\beta}\right),
\end{aligned}\right.
$$

then the gradient takes the form

$$
\nabla v_{\beta}=\left(I+\delta p_{\beta} s_{\beta} \mathcal{R}\right)^{-1} \nabla_{\Gamma} v^{[\beta]}+p_{\beta}^{-1} \delta^{-1} \frac{\partial v^{[\beta]}}{\partial s_{\beta}} \mathbf{n} .
$$

The volume element on the thin shell $\Omega_{\delta, \beta}$ becomes

$$
d \Omega_{\delta, \beta}=p_{\beta} \delta \operatorname{det} J_{\delta, \beta} d \Gamma d s_{\beta},
$$

where $J_{\delta, \beta}:=I+p_{\beta} \delta s_{\beta} \mathcal{R}$. Let $u_{\beta}$ and $v_{\beta}$ be two regular functions defined on $\Omega_{\delta, \beta}$. From (3.1) and (3.2), we get the change of variables formula

$$
\begin{aligned}
\int_{\Omega_{\delta, \beta}} \nabla u_{\beta} \cdot \nabla v_{\beta} d \Omega_{\delta, \beta}= & p_{\beta} \delta \int_{\Omega^{\beta}} J_{\delta, \beta}^{-2} \nabla_{\Gamma} u^{[\beta]} \cdot \nabla_{\Gamma} v^{[\beta]} \operatorname{det} J_{\delta, \beta} d \Gamma d s_{\beta} \\
& +p_{\beta}^{-1} \delta^{-1} \int_{\Omega^{\beta}} \partial_{s_{\beta}} u^{[\beta]} \partial_{s_{\beta}} v^{[\beta]} \operatorname{det} J_{\delta, \beta} d \Gamma d s_{\beta} .
\end{aligned}
$$

Remark 1. For any function $u$ defined in a neighborhood of $\Gamma$, we denote, for convenience, by $u_{\mid \Gamma}$ the trace of $u$ on $\Gamma$ indifferently in local coordinates or in Cartesian coordinates.

\section{The Asymptotic Analysis}

This section is devoted to the asymptotic analysis of the solution of Problem (2.1). We show that the latter is equivalent to a variational equation from which we derive the asymptotic expansion of $u_{\delta}$. We give a hierarchy of variational equations needed to determine the terms of the expansion and we calculate the first two terms of the expansion.

Let $v_{d}$ be in $H^{1}\left(\Omega_{\delta}\right)$. We denote by $v_{d_{\beta}}$ its restriction to $\Omega_{\delta, \beta}$. Multiplying Equation

$$
-\operatorname{div}\left(\alpha_{\delta} \nabla u_{d, \delta}\right)=f_{\mid \Omega_{\delta}} \quad \text { in } \Omega_{\delta}
$$

by test functions $v_{d}$, using $(2.1 \mathrm{~g}),(2.1 \mathrm{i}),(2.1 \mathrm{k})$ and Green's formula, we get

$$
\begin{array}{r}
\left\langle\alpha_{i} \partial_{\mathbf{n}_{\delta, 1}} u_{\mathrm{int}, \delta \mid \Gamma_{\delta, 1}}, v_{d_{1} \mid \Gamma_{\delta, 1}}\right\rangle_{H^{-1 / 2}\left(\Gamma_{\delta, 1}\right) \times H^{1 / 2}\left(\Gamma_{\delta, 1}\right)}+\alpha_{\delta} \int_{\Omega_{\delta}} \nabla u_{d, \delta} . \nabla v_{d} d \Omega_{\delta} \\
-\left\langle\alpha_{e} \partial_{\mathbf{n}_{\delta, 2}} u_{\mathrm{ext}, \delta \mid \Gamma_{\delta, 2}}, v_{d_{2} \mid \Gamma_{\delta, 2}}\right\rangle_{H^{-1 / 2}\left(\Gamma_{\delta, 2}\right) \times H^{1 / 2}\left(\Gamma_{\delta, 2}\right)}=\int_{\Omega_{\delta}} f_{\mid \Omega_{\delta}} v_{d} d \Omega_{\delta}
\end{array}
$$


in which $\langle., .\rangle_{H^{-1 / 2}\left(\Gamma_{\delta, \beta}\right) \times H^{1 / 2}\left(\Gamma_{\delta, \beta}\right)}$ denotes the duality pairing between $H^{-1 / 2}\left(\Gamma_{\delta, \beta}\right)$ and $H^{1 / 2}\left(\Gamma_{\delta, \beta}\right)$. We use the dilation in the thin layer and Formula (3.3), to obtain

$$
\begin{aligned}
& \left\langle\alpha_{i} \partial_{\mathbf{n}_{\delta, 1}} u_{\text {int }, \delta \mid \Gamma_{\delta, 1}} \circ \Phi_{1}(m,-1), v_{d}^{[1]}(m,-1)\right\rangle_{H^{-1 / 2}(\Gamma \times\{-1\}) \times H^{1 / 2}(\Gamma \times\{-1\})} \\
& -\left\langle\alpha_{e} \partial_{\mathbf{n}_{\delta, 2}} u_{\mathrm{ext}, \delta \mid \Gamma_{\delta, 2}} \circ \Phi_{2}(m, 1), v_{d}^{[2]}(m, 1)\right\rangle_{H^{-1 / 2}(\Gamma \times\{1\}) \times H^{1 / 2}(\Gamma \times\{1\})} \\
& \quad+\sum_{\beta=1}^{2}\left[\alpha_{\delta} \delta a_{\delta}^{[\beta]}\left(u_{d, \delta}^{[\beta]}, v_{d}^{[\beta]}\right)\right]=\int_{\Omega_{\delta}} f_{\mid \Omega_{\delta}} v_{d} d \Omega_{\delta},
\end{aligned}
$$

which is the starting point for the asymptotic analysis, where the bilinear form $a_{\delta}^{[\beta]}(.,$.$) is defined by$

$$
\begin{aligned}
a_{\delta}^{[\beta]}\left(u^{[\beta]}, v^{[\beta]}\right):= & p_{\beta} \int_{\Omega^{\beta}} J_{\delta, \beta}^{-2} \nabla_{\Gamma} u^{[\beta]} \cdot \nabla_{\Gamma} v^{[\beta]} \operatorname{det} J_{\delta, \beta} d \Gamma d s_{\beta} \\
& +p_{\beta}^{-1} \delta^{-2} \int_{\Omega^{\beta}} \partial_{s_{\beta}} u^{[\beta]} \partial_{s_{\beta}} v^{[\beta]} \operatorname{det} J_{\delta, \beta} d \Gamma d s_{\beta}
\end{aligned}
$$

for every $u^{[\beta]}$ and $v^{[\beta]}$ in $H^{1}\left(\Omega^{\beta}\right)$.

\subsection{Hierarchy of the variational equations}

In the spirit of $[21,25]$, we will consider two asymptotic expansions. Exterior expansions corresponding to the asymptotic expansion of $u_{\delta}$ restricted to $\Omega_{\text {ext }, \delta}$ and to $\Omega_{\mathrm{int}, \delta}$ characterized by the ansatz

$$
\begin{aligned}
& u_{\mathrm{ext}, \delta}=u_{\mathrm{ext}, 0}+\delta u_{\mathrm{ext}, 1}+\cdots, \\
& u_{\mathrm{int}, \delta}=u_{\mathrm{int}, 0}+\delta u_{\mathrm{int}, 1}+\cdots,
\end{aligned}
$$

in which the terms $u_{\text {int, } n}$ and $u_{\text {ext, } n}(n \in \mathbb{N})$ are independent of $\delta$ and defined on $\Omega_{\text {int }}$, where $\Omega_{\text {int }}$ is a bounded domain of $\mathbb{R}^{P}(P=2,3)$ with boundary $\Gamma$ (see Fig. 2) and on $\Omega_{\text {ext }}:=\Omega \backslash \bar{\Omega}_{\text {int }}$. They are respectively the limits of $\Omega_{\text {int }, \delta}$ and $\Omega_{\mathrm{ext}, \delta}$ for $\delta \rightarrow 0$. They fulfill

$$
\begin{cases}-\operatorname{div}\left(\alpha_{i} \nabla u_{\mathrm{int}, n}\right)=\delta_{0, n} f_{\mid \Omega_{\mathrm{int}}} & \text { in } \Omega_{\mathrm{int}}, \\ -\operatorname{div}\left(\alpha_{e} \nabla u_{\mathrm{ext}, n}\right)=\delta_{0, n} f_{\mid \Omega_{\mathrm{ext}}} & \text { in } \Omega_{\mathrm{ext}} \\ u_{\mathrm{ext}, n \mid \partial \Omega}=0 & \text { on } \partial \Omega\end{cases}
$$

where $\delta_{0, n}$ indicates the Kronecker symbol. And an interior expansion corresponding to the asymptotic expansion of $u_{d_{\beta}, \delta}$ written in a fixed domain and defined by the ansatz

$$
u_{d, \delta}^{[\beta]}=u_{0}^{[\beta]}+\delta u_{1}^{[\beta]}+\cdots, \quad \text { in } \Omega^{\beta},
$$


where the terms $u_{n}^{[\beta]}, n \in \mathbb{N}$, are independent of $\delta$. Using a Taylor expansion in the normal variable, we infer formally

$$
\begin{aligned}
\left(\sum_{n \geq 0} \delta^{n} u_{\mathrm{int}, n}\right) \circ \Phi_{1}\left(m, s_{1}\right) & =u_{\mathrm{int}, 0 \mid \Gamma}+\delta\left(u_{\mathrm{int}, 1 \mid \Gamma}+s_{1} p_{1} \partial_{\mathbf{n}} u_{\mathrm{int}, 0 \mid \Gamma}\right)+\cdots \\
& :=U_{\mathrm{int}, 0}+\delta U_{\mathrm{int}, 1}+\delta^{2} U_{\mathrm{int}, 2}+\cdots \\
\left(\sum_{n \geq 0} \delta^{n} u_{\mathrm{ext}, n}\right) \circ \Phi_{2}\left(m, s_{2}\right) & =u_{\mathrm{ext}, 0 \mid \Gamma}+\delta\left(u_{\mathrm{ext}, 1 \mid \Gamma}+s_{2} p_{2} \partial_{\mathbf{n}} u_{\mathrm{ext}, 0 \mid \Gamma}\right)+\cdots \\
: & =U_{\mathrm{ext}, 0}+\delta U_{\mathrm{ext}, 1}+\delta^{2} U_{\mathrm{ext}, 2}+\cdots
\end{aligned}
$$

Transmission Conditions (2.1f), (2.1h) and (2.1j) become

$$
\begin{aligned}
& u_{\mathrm{ext}, 0 \mid \Gamma}+\delta\left(u_{\mathrm{ext}, 1 \mid \Gamma}+p_{2} \partial_{\mathbf{n}} u_{\mathrm{ext}, 0 \mid \Gamma}\right)+\cdots=u_{0 \mid s_{2}=1}^{[2]}+\delta u_{1 \mid s_{2}=1}^{[2]}+\cdots, \\
& u_{0 \mid s_{1}=0}^{[1]}+\delta u_{1 \mid s_{1}=0}^{[1]}+\cdots=u_{0 \mid s_{2}=0}^{[2]}+\delta u_{1 \mid s_{2}=0}^{[2]}+\cdots, \\
& u_{\mathrm{int}, 0 \mid \Gamma}+\delta\left(u_{\mathrm{int}, 1 \mid \Gamma}-p_{1} \partial_{\mathbf{n}} u_{\mathrm{int}, 0 \mid \Gamma}\right)+\cdots=u_{0 \mid s_{1}=-1}^{[1]}+\delta u_{1 \mid s_{1}=-1}^{[1]}+\cdots
\end{aligned}
$$

Inserting (4.3) in the second equation of (4.5) and using Green's formula in $\Omega_{\delta, 2}$, we get

$$
\begin{aligned}
& \left\langle\alpha_{e} \partial_{\mathbf{n}}\left(\sum_{n \geq 0} \delta^{n} u_{\mathrm{ext}, n \mid \Gamma}\right), v_{d_{2} \mid \Gamma}\right\rangle_{H^{-1 / 2}(\Gamma) \times H^{1 / 2}(\Gamma)} \\
& \quad-\left\langle\alpha_{e} \partial_{\mathbf{n}_{\delta, 2}}\left(\sum_{n \geq 0} \delta^{n} u_{\mathrm{ext}, n \mid \Gamma_{\delta, 2}}\right), v_{d_{2} \mid \Gamma_{\delta, 2}}\right\rangle_{H^{-1 / 2}\left(\Gamma_{\delta, 1}\right) \times H^{1 / 2}\left(\Gamma_{\delta, 1}\right)} \\
& \quad+\alpha_{e} \int_{\Omega_{\delta, 2}} \nabla\left(\sum_{n \geq 0} \delta^{n} u_{\mathrm{ext}, n}\right) \cdot \nabla v_{d_{2}} d \Omega_{\delta, 2}=\int_{\Omega_{\delta, 2}} f_{\mid \Omega_{\delta, 2}} v_{d_{2}} d \Omega_{\delta, 2} .
\end{aligned}
$$

Using the scaling $s_{2}=\eta_{2} / \delta$, we obtain

$$
\begin{aligned}
& \int_{\Gamma} \alpha_{e} \partial_{\mathbf{n}}\left(\sum_{n \geq 0} \delta^{n} u_{\mathrm{ext}, n \mid \Gamma}\right) v_{d}^{[2]}(m, 0) d \Gamma+\alpha_{e} \delta a_{\delta}^{[2]}\left(\sum_{n \geq 0} \delta^{n} U_{\mathrm{ext}, n}, v_{d}^{[2]}\right) \\
& -\left\langle\alpha_{e} \partial_{\mathbf{n}_{\delta, 2}}\left(\sum_{n \geq 0} \delta^{n} u_{\mathrm{ext}, n \mid \Gamma \delta, 2}\right) \circ \Phi_{2}(m, 1), v_{d}^{[2]}(m, 1)\right\rangle_{H^{-1 / 2}(\Gamma \times\{1\}) \times H^{1 / 2}(\Gamma \times\{1\})} \\
& =\int_{\Omega_{\delta, 2}} f_{\mid \Omega_{\delta, 2}} v_{d_{2}} d \Omega_{\delta, 2} .
\end{aligned}
$$

In the same way, we obtain the equation for $\alpha_{i} \partial_{\mathbf{n}_{\delta, 1}}\left(\sum_{n \geq 0} \delta^{n} u_{\text {int }, n \mid \Gamma_{\delta, 1}}\right) \circ$ $\Phi_{1}(m,-1)$

$$
\begin{aligned}
& \alpha_{i} \delta a_{\delta}^{[1]}\left(\sum_{n \geq 0} \delta^{n} U_{\mathrm{int}, n}, v_{d}^{[1]}\right)-\int_{\Gamma} \alpha_{i} \partial_{\mathbf{n}}\left(\sum_{n \geq 0} \delta^{n} u_{\mathrm{int}, n \mid \Gamma}\right) v_{d}^{[1]}(m, 0) d \Gamma \\
& +\left\langle\alpha_{i} \partial_{\mathbf{n}_{\delta, 1}}\left(\sum_{n \geq 0} \delta^{n} u_{\mathrm{int}, n \mid \Gamma_{\delta, 1}}\right) \circ \Phi_{1}(m,-1),\right.
\end{aligned}
$$




$$
\left.v_{d}^{[1]}(m,-1)\right\rangle_{H^{-1 / 2}(\Gamma \times\{-1\}) \times H^{1 / 2}(\Gamma \times\{-1\})}=\int_{\Omega_{\delta, 1}} f_{\mid \Omega_{\delta, 1}} v_{d_{1}} d \Omega_{\delta, 1} .
$$

Inserting expansions (4.3), (4.4) and (4.6) in (4.1), using (4.7)-(4.8) and (4.12)(4.13), we get

$$
\begin{aligned}
& \int_{\Gamma} \alpha_{i} \partial_{\mathbf{n}}\left(\sum_{n \geq 0} \delta^{n} u_{\mathrm{int}, n \mid \Gamma}\right) v_{d}^{[1]}(m, 0) d \Gamma-\alpha_{i} \delta a_{\delta}^{[1]}\left(\sum_{n \geq 0} \delta^{n} U_{\mathrm{int}, n}, v_{d}^{[1]}\right) \\
& +\sum_{\beta=1}^{2}\left[\alpha_{\delta} \delta a_{\delta}^{[\beta]}\left(\sum_{n \geq 0} \delta^{n} u_{n}^{[\beta]}, v_{d}^{[\beta]}\right)\right]-\alpha_{e} \delta a_{\delta}^{[2]}\left(\sum_{n \geq 0} \delta^{n} U_{\mathrm{ext}, n}, v_{d}^{[2]}\right) \\
& -\int_{\Gamma} \alpha_{e} \partial_{\mathbf{n}}\left(\sum_{n \geq 0} \delta^{n} u_{\mathrm{ext}, n \mid \Gamma}\right) v_{d}^{[2]}(m, 0) d \Gamma=0 .
\end{aligned}
$$

Now, we use the identity (see [3, p. 1680])

$$
\begin{aligned}
J_{\delta, \beta}^{-2}:= & I-2 s_{\beta} p_{\beta} \delta \mathcal{R}+3\left(p_{\beta} s_{\beta} \delta \mathcal{R}\right)^{2}+\cdots+n\left(-p_{\beta} s_{\beta} \delta \mathcal{R}\right)^{n-1} \\
& +\left(-s_{\beta} p_{\beta} \delta \mathcal{R}\right)^{n}\left[n J_{\delta, \beta}^{-1}+J_{\delta, \beta}^{-2}\right] .
\end{aligned}
$$

Since

$$
\operatorname{det} J_{\delta, \beta}=1+2 p_{\beta} s_{\beta} \delta \mathcal{H}+\left(p_{\beta} s_{\beta} \delta\right)^{2} \mathcal{K},
$$

where $2 \mathcal{H}:=\operatorname{tr} \mathcal{R}$ and $\mathcal{K}:=\operatorname{det} \mathcal{R}$ are respectively the mean and the Gaussian curvatures of the surface $\Gamma$, the bilinear form $a_{\delta}^{[\beta]}(.,$.$) admits the expansion$

$$
\begin{aligned}
a_{\delta}^{[\beta]}(., .)= & \delta^{-2} a_{0,2}^{[\beta]}+\delta^{-1} a_{1,2}^{[\beta]}+\left(a_{2,2}^{[\beta]}+a_{0,1}^{[\beta]}\right)+\delta a_{1,1}^{[\beta]}+\cdots \\
& +\delta^{n-1} a_{n-1,1}^{[\beta]}+\delta^{n} r_{n}^{[\beta]}(\delta ; ., .),
\end{aligned}
$$

where the forms $a_{k, l}^{[\beta]}$ are independent of $\delta$ and are given by

$$
\begin{aligned}
& a_{0,2}^{[\beta]}\left(u^{[\beta]}, v^{[\beta]}\right):=\int_{\Omega^{\beta}} p_{\beta}^{-1} \partial_{s_{\beta}} u^{[\beta]} \partial_{s_{\beta}} v^{[\beta]} d \Gamma d s_{\beta}, \\
& a_{1,2}^{[\beta]}\left(u^{[\beta]}, v^{[\beta]}\right):=\int_{\Omega^{\beta}} 2 \mathcal{H} s_{\beta} \partial_{s_{\beta}} u^{[\beta]} \partial_{s_{\beta}} v^{[\beta]} d \Gamma d s_{\beta}, \\
& a_{2,2}^{[\beta]}\left(u^{[\beta]}, v^{[\beta]}\right):=\int_{\Omega^{\beta}} p_{\beta} \mathcal{K} s_{\beta}^{2} \partial_{s_{\beta}} u^{[\beta]} \partial_{s_{\beta}} v^{[\beta]} d \Gamma d s_{\beta}, \\
& a_{0,1}^{[\beta]}\left(u^{[\beta]}, v^{[\beta]}\right):=\int_{\Omega^{\beta}} p_{\beta} \nabla_{\Gamma} u^{[\beta]} \cdot \nabla_{\Gamma} v^{[\beta]} d \Gamma d s_{\beta}, \\
& a_{1,1}^{[\beta]}\left(u^{[\beta]}, v^{[\beta]}\right):=\int_{\Omega^{\beta}} 2 p_{\beta}^{2} s_{\beta}(\mathcal{H} I-\mathcal{R}) \nabla_{\Gamma} u^{[\beta]} \cdot \nabla_{\Gamma} v^{[\beta]} d \Gamma d s_{\beta}, \\
& a_{2,1}^{[\beta]}\left(u^{[\beta]}, v^{[\beta]}\right):=\int_{\Omega^{\beta}} p_{\beta}^{3}\left(\mathcal{K} I-4 \mathcal{H} \mathcal{R}+3 \mathcal{R}^{2}\right) s_{\beta}^{2} \nabla_{\Gamma} u^{[\beta]} \cdot \nabla_{\Gamma} v^{[\beta]} d \Gamma d s_{\beta}, \\
& a_{n-1,1}^{[\beta]}\left(u^{[\beta]}, v^{[\beta]}\right):=\int_{\Omega^{\beta}} p_{\beta}^{n}\left[(n-2) \mathcal{K} \mathcal{R}^{n-3}-(n-1) 2 \mathcal{H} \mathcal{R}^{n-2}\right. \\
&\left.+n \mathcal{R}^{n-1}\right]\left(-s_{\beta}\right)^{n-1} \nabla_{\Gamma} u^{[\beta]} \cdot \nabla_{\Gamma} v^{[\beta]} d \Gamma d s_{\beta}, \quad n>3 .
\end{aligned}
$$


The form $r_{n}^{[\beta]}(\delta ; .,$.$) is the remainder of Expansion (4.15) and is expressed by$ $r_{n}^{[\beta]}\left(\delta ; u^{[\beta]}, v^{[\beta]}\right):=\int_{\Omega^{\beta}}\left(B_{n, \delta}+2 \mathcal{H} B_{n-1, \delta}+\mathcal{K} B_{n-2, \delta}\right) s_{\beta}^{n} \nabla_{\Gamma} u^{[\beta]} \cdot \nabla_{\Gamma} v^{[\beta]} d \Gamma d s_{\beta}$

with

$$
B_{n, \delta}:= \begin{cases}(-\mathcal{R})^{n}\left(n J_{\delta, \beta}^{-1}+J_{\delta, \beta}^{-2}\right) & \text { if } n \geq 0 \\ J_{\delta, \beta}^{-2} & \text { otherwise. }\end{cases}
$$

Inserting Expansion (4.15) in (4.14) and matching the same powers of $\delta$, we obtain the following variational equations

$$
\begin{aligned}
& a_{0,2}^{[1]}\left(\alpha_{\delta} u_{0}^{[1]}-\alpha_{i} U_{\text {int }, 0}, v^{[1]}\right)+a_{0,2}^{[2]}\left(\alpha_{\delta} u_{0}^{[2]}-\alpha_{e} U_{\text {ext }, 0}, v^{[2]}\right)=0, \\
& a_{1,2}^{[1]}\left(\alpha_{\delta} u_{0}^{[1]}-\alpha_{i} U_{\text {int }, 0}, v^{[1]}\right)+a_{0,2}^{[1]}\left(\alpha_{\delta} u_{1}^{[1]}-\alpha_{i} U_{\text {int }, 1}, v^{[1]}\right) \\
& \quad+a_{1,2}^{[2]}\left(\alpha_{\delta} u_{0}^{[2]}-\alpha_{e} U_{\text {ext }, 0}, v^{[2]}\right)+a_{0,2}^{[2]}\left(\alpha_{\delta} u_{1}^{[2]}-\alpha_{e} U_{\text {ext }, 1}, v^{[2]}\right) \\
& =\alpha_{e} \int_{\Gamma} \partial_{\mathbf{n}} u_{\text {ext }, 0 \mid \Gamma} v^{[2]}(m, 0) d \Gamma-\alpha_{i} \int_{\Gamma} \partial_{\mathbf{n}} u_{\text {int }, 0 \mid \Gamma} v^{[1]}(m, 0) d \Gamma
\end{aligned}
$$

for all $v^{[\beta]}$ in $H^{1}\left(I_{\beta} ; L^{2}(\Gamma)\right)$ such that $v^{[1]}(., 0)=v^{[2]}(., 0)$,

$$
\begin{aligned}
& a_{0,2}^{[1]}\left(\alpha_{\delta} u_{2}^{[1]}-\alpha_{i} U_{\mathrm{int}, 2}, v^{[1]}\right)+a_{1,2}^{[1]}\left(\alpha_{\delta} u_{1}^{[1]}-\alpha_{i} U_{\mathrm{int}, 1}, v^{[1]}\right) \\
& +\left(a_{2,2}^{[1]}+a_{0,1}^{[1]}\right)\left(\alpha_{\delta} u_{0}^{[1]}-\alpha_{i} U_{\mathrm{int}, 0}, v^{[1]}\right) \\
& +a_{0,2}^{[2]}\left(\alpha_{\delta} u_{2}^{[2]}-\alpha_{e} U_{\text {ext }, 2}, v^{[2]}\right)+a_{1,2}^{[2]}\left(\alpha_{\delta} u_{1}^{[2]}-\alpha_{e} U_{\text {ext }, 1}, v^{[2]}\right) \\
& +\left(a_{2,2}^{[2]}+a_{0,1}^{[2]}\right)\left(\alpha_{\delta} u_{0}^{[2]}-\alpha_{e} U_{\text {ext }, 0}, v^{[2]}\right) \\
& =\alpha_{e} \int_{\Gamma} \partial_{\mathbf{n}} u_{\mathrm{ext}, 1 \mid \Gamma} v^{[2]}(m, 0) d \Gamma-\alpha_{i} \int_{\Gamma} \partial_{\mathbf{n}} u_{\mathrm{int}, 1 \mid \Gamma} v^{[1]}(m, 0) d \Gamma, \\
& a_{0,2}^{[1]}\left(\alpha_{\delta} u_{n+1}^{[1]}-\alpha_{i} U_{\mathrm{int}, n+1}, v^{[1]}\right)+a_{1,2}^{[1]}\left(\alpha_{\delta} u_{n}^{[1]}-\alpha_{i} U_{\mathrm{int}, n}, v^{[1]}\right) \\
& +a_{2,2}^{[1]}\left(\alpha_{\delta} u_{n-1}^{[1]}-\alpha_{i} U_{\text {int }, n-1}, v^{[1]}\right)+a_{0,1}^{[1]}\left(\alpha_{\delta} u_{n-1}^{[1]}-\alpha_{i} U_{\text {int }, n-1}, v^{[1]}\right) \\
& +a_{1,1}^{[1]}\left(\alpha_{\delta} u_{n-2}^{[1]}-\alpha_{i} U_{\text {int }, n-2}, v^{[1]}\right)+a_{2,1}^{[1]}\left(\alpha_{\delta} u_{n-3}^{[1]}-\alpha_{i} U_{\text {int }, n-3}, v^{[1]}\right) \\
& +\sum_{l=4}^{n} a_{l-1,1}^{[1]}\left(\alpha_{\delta} u_{n-l}^{[1]}-\alpha_{i} U_{\mathrm{int}, n-l}, v^{[1]}\right)+a_{0,2}^{[2]}\left(\alpha_{\delta} u_{n+1}^{[2]}-\alpha_{e} U_{\mathrm{ext}, n+1}, v^{[2]}\right) \\
& +a_{1,2}^{[2]}\left(\alpha_{\delta} u_{n}^{[2]}-\alpha_{e} U_{\text {ext }, n}, v^{[2]}\right)+a_{2,2}^{[2]}\left(\alpha_{\delta} u_{n-1}^{[2]}-\alpha_{e} U_{\text {ext }, n-1}, v^{[2]}\right) \\
& +a_{0,1}^{[2]}\left(\alpha_{\delta} u_{n-1}^{[2]}-\alpha_{e} U_{\text {ext }, n-1}, v^{[2]}\right)+a_{1,1}^{[2]}\left(\alpha_{\delta} u_{n-2}^{[2]}-\alpha_{e} U_{\text {ext }, n-2}, v^{[2]}\right) \\
& +a_{2,1}^{[2]}\left(\alpha_{\delta} u_{n-3}^{[2]}-\alpha_{e} U_{\text {ext }, n-3}, v^{[2]}\right)+\sum_{l=4}^{n} a_{l-1,1}^{[2]}\left(\alpha_{\delta} u_{n-l}^{[2]}-\alpha_{e} U_{\text {ext }, n-l}, v^{[2]}\right) \\
& =\alpha_{e} \int_{\Gamma} \partial_{\mathbf{n}} u_{\mathrm{ext}, n \mid \Gamma} v^{[2]}(m, 0) d \Gamma-\alpha_{i} \int_{\Gamma} \partial_{\mathbf{n}} u_{\mathrm{int}, n \mid \Gamma} v^{[1]}(m, 0) d \Gamma, \quad n \geq 4
\end{aligned}
$$

for all $v^{[\beta]}$ in $H^{1}\left(\Gamma \times I_{\beta}\right)$ such that $v^{[1]}(., 0)=v^{[2]}(., 0)$. 


\subsection{Calculation of the first terms}

In this paragraph, we first recall some theoretical results needed for our calculation. After this, we calculate explicitly the first two terms of Expansions (4.3)-(4.4) and (4.6) in order to present a recursive method to define successively the terms of these expansions. We need the following theorem.

Theorem 2. Let $F_{\text {int }} \in L^{2}\left(\Omega_{\text {int }}\right), F_{\text {ext }} \in L^{2}\left(\Omega_{\text {ext }}\right), h \in H^{1 / 2}(\Gamma)$ and $\zeta \in$ $H^{-1 / 2}(\Gamma)$. Then the following problem

$$
\begin{cases}-\operatorname{div}\left(\alpha_{i} \nabla U_{\text {int }}\right)=F_{\text {int }} & \text { in } \Omega_{\text {int }}, \\ -\operatorname{div}\left(\alpha_{e} \nabla U_{\text {ext }}\right)=F_{\text {ext }} & \text { in } \Omega_{\text {ext }}, \\ U_{\text {int } \mid \Gamma}-U_{\text {ext } \mid \Gamma}=h & \text { on } \Gamma, \\ \alpha_{i} \partial_{\mathbf{n}} U_{i n t \mid \Gamma}-\alpha_{e} \partial_{\mathbf{n}} U_{\text {ext } \mid \Gamma}=\zeta & \text { on } \Gamma, \\ U_{\text {ext } \mid \partial \Omega}=0 & \text { on } \partial \Omega\end{cases}
$$

admits a unique solution $\left(U_{\text {int }}, U_{\text {ext }}\right)$ in $H^{1}\left(\Omega_{\text {int }}\right) \times H^{1}\left(\Omega_{\text {ext }}\right)$. Moreover, for $k_{0} \in \mathbb{N}, F_{\text {int }} \in H^{k_{0}-2}\left(\Omega_{\text {int }}\right), F_{\text {ext }} \in H^{k_{0}-2}\left(\Omega_{\text {ext }}\right), h \in H^{k_{0}-1 / 2}(\Gamma), \zeta \in$ $H^{k_{0}-3 / 2}(\Gamma)$ and $\Gamma \cup \partial \Omega \mathcal{C}^{k_{0}}$-continuous, let $\left(U_{\text {int }}, U_{\text {ext }}\right) \in H^{1}\left(\Omega_{\text {int }}\right) \times H^{1}\left(\Omega_{\text {ext }}\right)$ be the solution of (4.20). For any positive integer $k \leq k_{0}$, there exists a constant $c_{k}$ such that

$$
\begin{aligned}
\left\|U_{i n t}\right\|_{H^{k}\left(\Omega_{i n t}\right)}+\left\|U_{e x t}\right\|_{H^{k}\left(\Omega_{e x t}\right)} \leq & c_{k}\left(\left\|F_{i n t}\right\|_{H^{k-2}\left(\Omega_{i n t}\right)}+\left\|F_{e x t}\right\|_{H^{k-2}\left(\Omega_{e x t}\right)}\right. \\
& \left.+\|h\|_{H^{k-1 / 2}(\Gamma)}+\|\zeta\|_{H^{k-3 / 2}(\Gamma)}\right) .
\end{aligned}
$$

Proof. Applying the techniques of Lemma 2.6 and Proposition 2.8 in [24, p. 37] we get the statement of theorem.

We also need the following technical lemma. This construction was motivated by [3] and its proof is not difficult.

Lemma 1. For $\beta=1,2$, let $k^{[\beta]}$ be a vectorial function in $L^{2}\left(\Omega^{\beta}, \mathbb{C}^{P}\right)$ such that the partial application $s_{\beta} \rightarrow k^{[\beta]}\left(., s_{\beta}\right)$ is valued in the space of vectorial fields tangent to $\Gamma$ and also $\operatorname{div}_{\Gamma} k^{[\beta]} \in L^{2}\left(\Omega^{\beta}\right)$. Then the solution $h^{[\beta]}$ of the variational equation

$$
\begin{gathered}
\mathcal{L}^{[\beta]} v^{[\beta]}:=\int_{\Omega^{\beta}} h^{[\beta]} \partial_{s_{\beta}} v^{[\beta]} d \Gamma d s_{\beta}+\int_{\Omega^{\beta}} k^{[\beta]} \cdot \nabla_{\Gamma} v^{[\beta]} d \Gamma d s_{\beta}=0 \\
\forall v^{[\beta]} \in H^{1}\left(\Omega^{\beta}\right), \quad v^{[\beta]}(., 0)=0
\end{gathered}
$$

is explicitly given by

$$
h^{[\beta]}\left(m, s_{\beta}\right)=\int_{s_{\beta}}^{(-1)^{\beta}} \operatorname{div}_{\Gamma} k^{[\beta]}(m, \lambda) d \lambda .
$$

Moreover, if $v^{[\beta]}(., 0) \neq 0$, we have

$$
\begin{aligned}
\mathcal{L}^{[\beta]} v^{[\beta]} & =(-1)^{\beta+1} \int_{\Gamma} h^{[\beta]}(m, 0) v^{[\beta]}(m, 0) d \Gamma \\
& =\int_{\Gamma}\left[(-1)^{\beta+1} \int_{0}^{(-1)^{\beta}} \operatorname{div}_{\Gamma} k^{[\beta]}\left(m, s_{\beta}\right) d s_{\beta}\right] v^{[\beta]}(m, 0) d \Gamma .
\end{aligned}
$$




\subsubsection{Term of order 0}

The choice of $v^{[2]}=0$ in (4.16) gives

$$
a_{0,2}^{[1]}\left(\alpha_{\delta} u_{0}^{[1]}-\alpha_{i} U_{\mathrm{int}, 0}, v^{[1]}\right)=0 .
$$

Applying Lemma 1 with

$$
h^{[1]}=p_{1}^{-1} \alpha_{\delta} \partial_{s_{1}} u_{0}^{[1]}-p_{1}^{-1} \alpha_{i} \partial_{s_{1}} U_{\text {int }, 0}=p_{1}^{-1} \alpha_{\delta} \partial_{s_{1}} u_{0}^{[1]}, \quad k^{[1]}=0,
$$

we obtain $\partial_{s_{1}} u_{0}^{[1]}=0$. Similarly, the choice of $v^{[1]}=0$ in (4.16) gives

$$
a_{0,2}^{[2]}\left(\alpha_{\delta} u_{0}^{[2]}-\alpha_{e} U_{\mathrm{ext}, 0}, v^{[2]}\right)=0 .
$$

We apply Lemma 1 with

$$
h^{[2]}=p_{2}^{-1} \alpha_{\delta} \partial_{s_{2}} u_{0}^{[2]}-p_{2}^{-1} \alpha_{e} \partial_{s_{2}} U_{\text {ext }, 0}=p_{2}^{-1} \alpha_{\delta} \partial_{s_{2}} u_{0}^{[2]}, \quad k^{[2]}=0
$$

to obtain $\partial_{s_{2}} u_{0}^{[2]}=0$. Using (4.9), (4.10) and (4.11), we get

$$
u_{\text {int }, 0 \mid \Gamma}=u_{0}^{[1]}\left(m, s_{1}\right)=u_{0}^{[2]}\left(m, s_{2}\right)=u_{\mathrm{ext}, 0 \mid \Gamma}, \quad m \in \Gamma, \forall\left(m, s_{\beta}\right) \in \Omega^{\beta} .
$$

In the same manner as above, using Lemma 1 and (4.17), we find

$$
\begin{gathered}
p_{1}^{-1} \alpha_{\delta} \partial_{s_{1}} u_{1}^{[1]}-\alpha_{i} \partial_{\mathbf{n}} u_{\text {int }, 0 \mid \Gamma}=0, \\
p_{2}^{-1} \alpha_{\delta} \partial_{s_{2}} u_{1}^{[2]}-\alpha_{e} \partial_{\mathbf{n}} u_{\text {ext }, 0 \mid \Gamma}=0 .
\end{gathered}
$$

Moreover, for all $v^{[\beta]}$, we have

$$
\alpha_{i} \int_{\Gamma} \partial_{\mathbf{n}} u_{\mathrm{int}, 0 \mid \Gamma} v^{[1]}(m, 0) d \Gamma=\alpha_{e} \int_{\Gamma} \partial_{\mathbf{n}} u_{\mathrm{ext}, 0 \mid \Gamma} v^{[2]}(m, 0) d \Gamma .
$$

So

$$
\alpha_{i} \partial_{\mathbf{n}} u_{\text {int }, 0 \mid \Gamma}=\alpha_{e} \partial_{\mathbf{n}} u_{\text {ext }, 0 \mid \Gamma} .
$$

Let us define $\alpha_{0}$ and $u_{n}$ by

$$
\alpha_{0}(x):=\left\{\begin{array}{ll}
\alpha_{e} & \text { if } x \in \Omega_{\mathrm{ext}}, \\
\alpha_{i} & \text { if } x \in \Omega_{\mathrm{int}}
\end{array} \quad \text { and } \quad u_{n}:= \begin{cases}u_{\mathrm{ext}, n} & \text { in } \Omega_{\mathrm{ext}} \\
u_{\mathrm{int}, n} & \text { in } \Omega_{\mathrm{int}}\end{cases}\right.
$$

Therefore, with (4.5), (4.21), (4.24) and Theorem $2, u_{0}$ is the unique solution of the following problem

$$
\begin{cases}-\operatorname{div}\left(\alpha_{0} \nabla u_{0}\right)=f & \text { in } \Omega \\ u_{0 \mid \partial \Omega}=0 & \text { on } \partial \Omega\end{cases}
$$

Note that $u_{0}$ is nothing but the solution of the initial problem without the thin layer. 


\subsubsection{Term of order 1}

Integrating Relations (4.22) and (4.23) in $s_{\beta}$ and identifying terms of order 1 in (4.9) and (4.11), yields

$$
\begin{aligned}
u_{1}^{[1]}\left(m, s_{1}\right) & =u_{1}^{[1]}(m,-1)+\left(s_{1}+1\right) p_{1} \alpha_{i} \alpha_{\delta}^{-1} \partial_{\mathbf{n}} u_{\mathrm{int}, 0 \mid \Gamma} \\
& =u_{\mathrm{int}, 1 \mid \Gamma}+p_{1}\left[\left(s_{1}+1\right) \alpha_{i} \alpha_{\delta}^{-1}-1\right] \partial_{\mathbf{n}} u_{\mathrm{int}, 0 \mid \Gamma}, \quad \forall\left(m, s_{1}\right) \in \Omega^{1}
\end{aligned}
$$

and

$$
\begin{aligned}
u_{1}^{[2]}\left(m, s_{2}\right) & =u_{1}^{[2]}(m, 1)+\left(s_{2}-1\right) p_{2} \alpha_{e} \alpha_{\delta}^{-1} \partial_{\mathbf{n}} u_{\mathrm{ext}, 0 \mid \Gamma} \\
& =u_{\mathrm{ext}, 1 \mid \Gamma}+p_{2}\left[\left(s_{2}-1\right) \alpha_{e} \alpha_{\delta}^{-1}+1\right] \partial_{\mathbf{n}} u_{\mathrm{ext}, 0 \mid \Gamma}, \quad \forall\left(m, s_{2}\right) \in \Omega^{2} .
\end{aligned}
$$

The identification of first order terms of (4.10) gives the first transmission condition on $\Gamma$

$$
u_{\mathrm{int}, 1 \mid \Gamma}-u_{\mathrm{ext}, 1 \mid \Gamma}=p_{1}\left(1-\alpha_{i} \alpha_{\delta}^{-1}\right) \partial_{\mathbf{n}} u_{\mathrm{int}, 0 \mid \Gamma}+p_{2}\left(1-\alpha_{e} \alpha_{\delta}^{-1}\right) \partial_{\mathbf{n}} u_{\mathrm{ext}, 0 \mid \Gamma}
$$

The second one follows the same lines as for order 0 . The choice of $v^{[2]}=0$ in (4.18) gives

$$
a_{0,2}^{[1]}\left(\alpha_{\delta} u_{2}^{[1]}-\alpha_{i} U_{\mathrm{int}, 2}, v^{[1]}\right)+a_{0,1}^{[1]}\left(\alpha_{\delta} u_{0}^{[1]}-\alpha_{i} U_{\mathrm{int}, 0}, v^{[1]}\right)=0 .
$$

We apply Lemma 1 with

$$
\begin{aligned}
& h^{[1]}=\frac{\alpha_{\delta}}{p_{1}} \partial_{s_{1}} u_{2}^{[1]}-\frac{\alpha_{i}}{p_{1}} \partial_{s_{1}} U_{\mathrm{int}, 2}=\frac{\alpha_{\delta}}{p_{1}} \partial_{s_{1}} u_{2}^{[1]}-\alpha_{i} \partial_{\mathbf{n}} u_{\mathrm{int}, 1 \mid \Gamma}-s_{1} p_{1} \alpha_{i} \partial_{\mathbf{n}}^{2} u_{\mathrm{int}, 0 \mid \Gamma}, \\
& k^{[1]}=p_{1} \nabla_{\Gamma}\left(\alpha_{\delta} u_{0}^{[1]}-\alpha_{i} U_{\mathrm{int}, 0}\right)=p_{1}\left(\alpha_{\delta}-\alpha_{i}\right) \nabla_{\Gamma} u_{\mathrm{int}, 0 \mid \Gamma}
\end{aligned}
$$

to find

$$
\begin{aligned}
& \frac{\alpha_{\delta}}{p_{1}} \partial_{s_{1}} u_{2}^{[1]}\left(m, s_{1}\right)-\alpha_{i} \partial_{\mathbf{n}} u_{\mathrm{int}, 1 \mid \Gamma}-s_{1} p_{1} \alpha_{i} \partial_{\mathbf{n}}^{2} u_{\mathrm{int}, 0 \mid \Gamma} \\
& \quad=-\left(s_{1}+1\right) p_{1}\left(\alpha_{\delta}-\alpha_{i}\right) \Delta_{\Gamma} u_{\mathrm{int}, 0 \mid \Gamma} .
\end{aligned}
$$

Moreover, for all $v^{[1]}$ we obtain

$$
\mathcal{L}^{[1]} v^{[1]}=-\int_{\Gamma} p_{1}\left(\alpha_{\delta}-\alpha_{1}\right) \Delta_{\Gamma} u_{\mathrm{int}, 0 \mid \Gamma} v^{[1]}(m, 0) d \Gamma .
$$

Similarly, the choice of $v^{[1]}=0$ in (4.18) gives

$$
a_{0,2}^{[2]}\left(\alpha_{\delta} u_{2}^{[2]}-\alpha_{e} U_{\text {ext }, 2}, v^{[2]}\right)+a_{0,1}^{[2]}\left(\alpha_{\delta} u_{0}^{[2]}-\alpha_{e} U_{\text {ext }, 0}, v^{[2]}\right)=0 .
$$

We apply Lemma 1 with

$$
\begin{aligned}
& h^{[2]}=\frac{\alpha_{\delta}}{p_{2}} \partial_{s_{2}} u_{2}^{[2]}-\frac{\alpha_{e}}{p_{2}} \partial_{s_{2}} U_{\text {ext }, 2}=\frac{\alpha_{\delta}}{p_{2}} \partial_{s_{2}} u_{2}^{[2]}-\alpha_{e} \partial_{\mathbf{n}} u_{\text {ext }, 1 \mid \Gamma}-s_{2} p_{2} \alpha_{e} \partial_{\mathbf{n}}^{2} u_{\text {ext }, 0 \mid \Gamma}, \\
& k^{[2]}=p_{2} \nabla_{\Gamma}\left(\alpha_{\delta} u_{0}^{[2]}-\alpha_{e} U_{\text {ext }, 0}\right)=p_{2}\left(\alpha_{\delta}-\alpha_{e}\right) \nabla_{\Gamma} u_{\text {ext }, 0 \mid \Gamma}
\end{aligned}
$$


to find

$$
\begin{aligned}
& \frac{\alpha_{\delta}}{p_{2}} \partial_{s_{2}} u_{2}^{[2]}\left(m, s_{2}\right)-\alpha_{e} \partial_{\mathbf{n}} u_{\mathrm{ext}, 1 \mid \Gamma}-s_{2} p_{2} \alpha_{e} \partial_{\mathbf{n}}^{2} u_{\mathrm{ext}, 0 \mid \Gamma} \\
& \quad=\left(1-s_{2}\right) p_{2}\left(\alpha_{\delta}-\alpha_{e}\right) \Delta_{\Gamma} u_{\mathrm{ext}, 0 \mid \Gamma} .
\end{aligned}
$$

Furthermore, for all $v^{[2]}$ we obtain

$$
\mathcal{L}^{[2]} v^{[2]}=-\int_{\Gamma} p_{2}\left(\alpha_{\delta}-\alpha_{e}\right) \Delta_{\Gamma} u_{\mathrm{ext}, 0 \mid \Gamma} v^{[2]}(m, 0) d \Gamma .
$$

As a consequence,

$$
\alpha_{i} \partial_{\mathbf{n}} u_{\mathrm{int}, 1 \mid \Gamma}-\alpha_{e} \partial_{\mathbf{n}} u_{\mathrm{ext}, 1 \mid \Gamma}=p_{1}\left(\alpha_{\delta}-\alpha_{i}\right) \Delta_{\Gamma} u_{\mathrm{int}, 0 \mid \Gamma}+p_{2}\left(\alpha_{\delta}-\alpha_{e}\right) \Delta_{\Gamma} u_{\mathrm{ext}, 0 \mid \Gamma}
$$

It follows from $(4.5),(4.25),(4.26)$ and Theorem 2 that $u_{1}$ is the unique solution of the following problem

$$
\begin{cases}-\operatorname{div}\left(\alpha_{i} \nabla u_{\mathrm{int}, 1}\right)=0 & \text { in } \Omega_{\mathrm{int}} \\ -\operatorname{div}\left(\alpha_{e} \nabla u_{\mathrm{ext}, 1}\right)=0 & \text { in } \Omega_{\text {ext }} \\ u_{\mathrm{ext}, 1 \mid \partial \Omega}=0 & \text { on } \partial \Omega\end{cases}
$$

with transmission conditions on $\Gamma$

$$
\begin{array}{r}
u_{\mathrm{int}, 1 \mid \Gamma}-u_{\mathrm{ext}, 1 \mid \Gamma}=p_{1}\left(1-\frac{\alpha_{i}}{\alpha_{\delta}}\right) \partial_{\mathbf{n}} u_{\mathrm{int}, 0 \mid \Gamma}+p_{2}\left(1-\frac{\alpha_{e}}{\alpha_{\delta}}\right) \partial_{\mathbf{n}} u_{\mathrm{ext}, 0 \mid \Gamma}, \\
\alpha_{i} \partial_{\mathbf{n}} u_{\mathrm{int}, 1 \mid \Gamma}-\alpha_{e} \partial_{\mathbf{n}} u_{\mathrm{ext}, 1 \mid \Gamma}=p_{1}\left(\alpha_{\delta}-\alpha_{i}\right) \Delta_{\Gamma} u_{\mathrm{int}, 0 \mid \Gamma}+p_{2}\left(\alpha_{\delta}-\alpha_{e}\right) \Delta_{\Gamma} u_{\mathrm{ext}, 0 \mid \Gamma}
\end{array}
$$

or

$$
\begin{aligned}
u_{\text {int }, 1 \mid \Gamma}-u_{\text {ext }, 1 \mid \Gamma} & =\left[p_{1}\left(1-\frac{\alpha_{i}}{\alpha_{\delta}}\right)+p_{2}\left(\frac{\alpha_{i}}{\alpha_{e}}-\frac{\alpha_{i}}{\alpha_{\delta}}\right)\right] \partial_{\mathbf{n}} u_{\text {int }, 0 \mid \Gamma}, \\
\alpha_{i} \partial_{\mathbf{n}} u_{\text {int }, 1 \mid \Gamma}-\alpha_{e} \partial_{\mathbf{n}} u_{\text {ext }, 1 \mid \Gamma} & =\left[p_{1}\left(\alpha_{\delta}-\alpha_{i}\right)+p_{2}\left(\alpha_{\delta}-\alpha_{e}\right)\right] \Delta_{\Gamma} u_{\text {int }, 0 \mid \Gamma} .
\end{aligned}
$$

Remark 2. The determination of the first two terms of the asymptotic expansion in the two-dimensional case does not differ from the case $N=3$.

\subsection{Existence and uniqueness of $\left(u_{\mathrm{int}, j}\right),\left(u_{\mathrm{ext}, j}\right)$ and $\left(u_{j}^{[\beta]}\right)$}

Theorem 3. The sequences $\left(u_{i n t, j}\right),\left(u_{e x t, j}\right)$ and $\left(u_{j}^{[\beta]}\right)$ exist and are unique. Furthermore, for any $k \in \mathbb{N}$ and $j \in \mathbb{N}$, we have $u_{\text {int }, j} \in H^{k}\left(\Omega_{\text {int }}\right), u_{\text {ext }, j} \in$ $H^{k}\left(\Omega_{\text {ext }}\right)$ and $u_{j}^{[\beta]} \in H^{k}\left(\Omega^{\beta}\right)$.

Proof. In the previous paragraphs, we have determined the first two terms of Expansions (4.3), (4.4), (4.6), and have shown, provided that $f$ is $\mathcal{C}^{\infty}$, that for $j=0,1$ and for any $k \in \mathbb{N}$, there exists a constant $c$ independent of $\delta$ such that $\left\|u_{\mathrm{int}, j}\right\|_{H^{k}\left(\Omega_{\mathrm{int}}\right)}+\left\|u_{\mathrm{ext}, j}\right\|_{H^{k}\left(\Omega_{\mathrm{ext}}\right)} \leq c$. Also, we have shown that $u_{j}^{[\beta]}$ is a polynomial of degree $j$ in $s_{\beta}$ and using the regularity of $\left(u_{\text {int }, j}\right)_{0 \leq j \leq 1}$ and $\left(u_{\text {ext }, j}\right)_{0 \leq j \leq 1}$, we deduce that $\left\|u_{j}^{[\beta]}\right\|_{H^{k}\left(\Omega^{\beta}\right)} \leq c$. Let us now present the general 
construction : Let us assume that the terms $u_{\text {int }, j}, u_{\text {ext }, j}$ and $u_{j}^{[\beta]}$ were built for $j<n$. The construction of $u_{\mathrm{int}, j}, u_{\mathrm{ext}, j}$ and $u_{j}^{[\beta]}$ consists of four steps.

Step 1. We define $\partial_{s_{\beta}} u_{n}^{[\beta]}$ from Lemma 1 and Problem (4.19) at rank $(n-1)$. Thus the jump condition $u_{\mathrm{int}, n \mid \Gamma}-u_{\mathrm{ext}, n \mid \Gamma}$ can be determined by the relation

$$
\begin{aligned}
u_{\text {int }, n \mid \Gamma}-u_{\text {ext }, n \mid \Gamma}:= & \sum_{j=1}^{n} \frac{1}{j !} p_{2}^{j} \partial_{\mathbf{n}}^{j} u_{\text {ext }, n-j \mid \Gamma}-\sum_{j=1}^{n} \frac{(-1)^{j}}{j !} p_{1}^{j} \partial_{\mathbf{n}}^{j} u_{\text {int }, n-j \mid \Gamma} \\
& -\int_{-1}^{0} \partial_{s_{1}} u_{n}^{[1]}\left(m, s_{1}\right) d s_{1}-\int_{0}^{1} \partial_{s_{2}} u_{n}^{[1]}\left(m, s_{2}\right) d s_{2} .
\end{aligned}
$$

Step 2. Lemma 1 and Problem (4.19) at rank $n$ determine the transmission condition $\alpha_{i} \partial_{\mathbf{n}} u_{\mathrm{int}, n \mid \Gamma}-\alpha_{e} \partial_{\mathbf{n}} u_{\mathrm{ext}, n \mid \Gamma}$ in terms of the surfacic divergence of $\partial_{s_{\beta}} u_{j}^{[\beta]}(j<n)$.

Step 3. Step 1, Step 2, (4.5) and Theorem 2 guarantee the existence, uniqueness and regularity of $u_{\mathrm{int}, n}$ and $u_{\mathrm{ext}, n}$.

Step 4. The determination of $u_{n}^{[\beta]}$ can be achieved by integrating $\partial_{s_{\beta}} u_{n}^{[\beta]}$, defined in Step 1, in $s_{\beta}$ and using transmission conditions (4.9) and (4.11). The smoothness of $u_{n}^{[\beta]}$ follows from the regularity of $\left(u_{\mathrm{int}, j}\right)_{j \leq n}$ and $\left(u_{\mathrm{ext}, j}\right)_{j \leq n}$.

\section{Convergence Theorem}

The process described in the previous section can be continued up to any order provided that the data are sufficiently regular. We can also estimate the error made by truncating the series after a finite number of terms. Let $n$ be in $\mathbb{N}$, we set

$$
\begin{aligned}
u_{\mathrm{int}, \delta}^{(n)} & :=\sum_{j=0}^{n} \delta^{j} u_{\mathrm{int}, j}, \quad u_{\mathrm{ext}, \delta}^{(n)}:=\sum_{j=0}^{n} \delta^{j} u_{\mathrm{ext}, j}, \\
u_{d, \delta}^{(n)}: & :=\left\{\begin{array}{l}
u_{d_{1}, \delta}^{(n)}:=\sum_{j=0}^{n} \delta^{j} u_{d_{1}, j} \text { in } \Omega_{\delta, 1,} \\
u_{d_{2}, \delta}^{(n)}:=\sum_{j=0}^{n} \delta^{j} u_{d_{2}, j} \text { in } \Omega_{\delta, 2},
\end{array}\right.
\end{aligned}
$$

where $u_{d_{\beta}, j}(x):=\widetilde{u}_{d_{\beta}, j}\left(m, \delta s_{\beta}\right):=u_{j}^{[\beta]}\left(m, s_{\beta}\right) ; \forall x=\Phi_{\beta}\left(m, s_{\beta}\right) \in \Omega_{\delta, \beta}$.

Theorem 4 [Convergence Theorem]. For all integers $n$, there exists a constant $c$ independent of $\delta$ such as

$$
\begin{aligned}
& \left\|u_{i n t, \delta}-u_{i n t, \delta}^{(n)}\right\|_{H^{1}\left(\Omega_{i n t, \delta}\right)}+\delta^{1 / 2}\left\|u_{d, \delta}-u_{d, \delta}^{(n)}\right\|_{H^{1}\left(\Omega_{\delta}\right)} \\
& +\left\|u_{e x t, \delta}-u_{e x t, \delta}^{(n)}\right\|_{H^{1}\left(\Omega_{e x t, \delta}\right)} \leq c \delta^{n+1}
\end{aligned}
$$

Proof. Since $f$ is $\mathcal{C}^{\infty}$, all terms in Expansions (4.3), (4.4) and (4.6) up to order $n+1$ may be obtained from Equations (4.16)-(4.19). Let us define the remainders $R_{D_{1}, n}, R_{D_{2}, n}, R_{1, n}$ and $R_{2, n}$ of Taylor expansions in the normal 
variable with respect to $\delta$ up to order $n$ of $u_{\mathrm{int}, \delta \mid \Gamma_{\delta, 1}}^{(n)}, u_{\mathrm{ext}, \delta \mid \Gamma_{\delta, 2}}^{(n)}, u_{\mathrm{int}, \delta}^{(n)}$ and $u_{\mathrm{ext}, \delta}^{(n)}$ respectively by

$$
\begin{aligned}
R_{D_{1}, n} & :=u_{\mathrm{int}, \delta \mid \Gamma_{\delta, 1}}^{(n)}-\sum_{j=0}^{n} \sum_{l=0}^{n-j} \frac{(-1)^{l} \delta^{j+l}}{l !} p_{1}^{l} \partial_{\mathbf{n}}^{l} u_{\mathrm{int}, j \mid \Gamma} \\
R_{D_{2}, n} & :=u_{\mathrm{ext}, \delta \mid \Gamma_{\delta, 2}}^{(n)}-\sum_{j=0}^{n} \sum_{l=0}^{n-j} \frac{\delta^{j+l}}{l !} p_{2}^{l} \partial_{\mathbf{n}}^{l} u_{\mathrm{ext}, j \mid \Gamma}, \\
R_{1, n}(x) & :=R_{1, n}^{[1]}\left(m, s_{1}\right):=\left(u_{\mathrm{int}, \delta}^{(n)}\right)^{[1]}-\sum_{j=0}^{n} \sum_{l=0}^{n-j} \frac{\left(s_{1}\right)^{l} \delta^{j+l}}{l !} p_{1}^{l} \partial_{\mathbf{n}}^{l} u_{\mathrm{int}, j \mid \Gamma} \\
& :=\left(u_{\mathrm{int}, \delta}^{(n)}\right)^{[1]}-\sum_{j=0}^{n} \delta^{j} U_{\mathrm{int}, j}, \quad \forall x=\Phi_{1}\left(m, s_{1}\right) \in \Omega_{\delta, 1}, \\
R_{2, n}(x) & :=R_{2, n}^{[2]}\left(m, s_{2}\right):=\left(u_{\mathrm{ext}, \delta}^{(n)}\right)^{[2]}-\sum_{j=0}^{n} \sum_{l=0}^{n-j} \frac{\left(s_{2}\right)^{l} \delta^{j+l}}{l !} p_{2}^{l} \partial_{\mathbf{n}}^{l} u_{\mathrm{ext}, j \mid \Gamma} \\
& :=\left(u_{\mathrm{ext}, \delta}^{(n)}\right)^{[2]}-\sum_{j=0}^{n} \delta^{j} U_{\mathrm{ext}, j}, \quad \forall x=\Phi_{2}\left(m, s_{2}\right) \in \Omega_{\delta, 2},
\end{aligned}
$$

where $s_{\beta} \in I_{\beta}$. We shall rely on the following proposition to show the estimates of the remainders $R_{D_{\beta}, n}$ and $R_{\beta, n}$. The steps of the proof are very similar to those given in [25, Section 5]. We refer the reader to this paper.

Proposition 1. There exists a constant $c>0$, independent of $\delta$, such as

$$
\left\|\nabla R_{\beta, n}\right\|_{L^{2}\left(\Omega_{\delta, \beta}\right)} \leq c \delta^{n+1 / 2}, \quad\left\|\nabla_{\Gamma}^{(j)} R_{D_{\beta}, n}\right\|_{L^{2}(\Gamma)} \leq c \delta^{n+1 / 2}, \quad \text { for } j=0,1 .
$$

Moreover, there exists an extension $\mathcal{P} R$ of $R_{D_{\beta}, n}$ into $\Omega_{\delta}$ with

$$
\|\mathcal{P} R\|_{H^{1}\left(\Omega_{\delta}\right)} \leq c \delta^{n}
$$

Continuation of the proof of Theorem 4. Let $r_{\mathrm{int}, \delta}^{n}, r_{d, \delta}^{n}$ and $r_{\mathrm{ext}, \delta}^{n}$ be the remainders made by truncating Series (4.3), (4.4) and (4.6)

$$
r_{\mathrm{int}, \delta}^{n}:=u_{\mathrm{int}, \delta}-u_{\mathrm{int}, \delta}^{(n)}, \quad r_{\mathrm{ext}, \delta}^{n}:=u_{\mathrm{ext}, \delta}-u_{\mathrm{ext}, \delta}^{(n)}, \quad r_{d, \delta}^{n}:=u_{d, \delta}-u_{d, \delta}^{(n)}
$$

and $\mathcal{L}_{\delta}$ be the linear form defined on $H_{0}^{1}(\Omega)$

$$
\begin{aligned}
\mathcal{L}_{\delta} v:= & \alpha_{i} \int_{\Omega_{\mathrm{int}, \delta}} \nabla r_{\mathrm{int}, \delta}^{n} \cdot \nabla v_{\mathrm{int}, \delta} d \Omega_{\mathrm{int}, \delta}+\alpha_{\delta} \int_{\Omega_{\delta}} \nabla\left(r_{d, \delta}^{n}-\mathcal{P} R\right) . \nabla v_{d} d \Omega_{\delta} \\
& +\alpha_{e} \int_{\Omega_{\mathrm{ext}, \delta}} \nabla r_{\mathrm{ext}, \delta}^{n} \cdot \nabla v_{\mathrm{ext}, \delta} d \Omega_{\mathrm{ext}, \delta},
\end{aligned}
$$

in which $\mathcal{P} R$ is the extension function of $R_{D_{\beta}, n}$ into $\Omega_{\delta}$ and $v_{\text {int }, \delta}, v_{d}$, and $v_{\text {ext }, \delta}$ are the restrictions of $v$ respectively to the domains $\Omega_{\text {int }, \delta}, \Omega_{\delta}$ and $\Omega_{\text {ext }, \delta}$. 
Using Green's formula in $\Omega_{\text {int }}$ and in $\Omega_{\text {ext }}$ with the help of (4.5), we obtain

$$
\begin{aligned}
\mathcal{L}_{\delta} v= & \int_{\Omega} f v d \Omega-\alpha_{i} \int_{\Omega_{\mathrm{int}}} \nabla u_{\mathrm{int}, \delta}^{(n)} \cdot \nabla v_{\mid \Omega_{\mathrm{int}}} d \Omega_{\mathrm{int}}+\alpha_{i} \int_{\Omega_{\delta, 1}} \nabla u_{\mathrm{int}, \delta}^{(n)} \cdot \nabla v_{d, 1} d \Omega_{\delta, 1} \\
& -\alpha_{\delta} \int_{\Omega_{\delta}} \nabla u_{d, \delta}^{(n)} \cdot \nabla v_{d} d \Omega_{\delta}-\alpha_{e} \int_{\Omega_{\mathrm{ext}}} \nabla u_{\mathrm{ext}, \delta}^{(n)} \cdot \nabla v_{\mid \Omega_{\mathrm{ext}}} d \Omega_{\mathrm{ext}} \\
& +\alpha_{e} \int_{\Omega_{\delta, 1}} \nabla u_{\mathrm{ext}, \delta}^{(n)} \cdot \nabla v_{d, 1} d \Omega_{\delta, 1}-\alpha_{\delta} \int_{\Omega_{\delta}} \nabla \mathcal{P} R \cdot \nabla v_{d} d \Omega_{\delta} \\
= & -\alpha_{i} \int_{\Gamma}\left(\partial_{\mathbf{n}} u_{\mathrm{int}, 0 \mid \Gamma}+\cdots+\delta^{n} \partial_{\mathbf{n}} u_{\mathrm{int}, n \mid \Gamma}\right) v_{d, 1 \mid \Gamma} d \Gamma \\
& +\alpha_{i} \int_{\Omega_{\delta, 1}} \nabla u_{\mathrm{int}, \delta}^{(n)} \cdot \nabla v_{d, 1} d \Omega_{\delta, 1}-\alpha_{\delta} \int_{\Omega_{\delta}} \nabla u_{d, \delta}^{(n)} \cdot \nabla v_{d} d \Omega_{\delta} \\
& +\alpha_{e} \int_{\Omega_{\delta, 2}} \nabla u_{\mathrm{ext}, \delta}^{(n)} \cdot \nabla v_{d, 2} d \Omega_{\delta, 2}-\alpha_{\delta} \int_{\Omega_{\delta}} \nabla \mathcal{P} R . \nabla v_{d} d \Omega_{\delta} \\
& +\alpha_{e} \int_{\Gamma}\left(\partial_{\mathbf{n}} u_{\mathrm{ext}, 0 \mid \Gamma}+\cdots+\delta^{n} \partial_{\mathbf{n}} u_{\mathrm{ext}, n \mid \Gamma}\right) v_{d, 2 \mid \Gamma} d \Gamma .
\end{aligned}
$$

It follows, from (5.1)-(5.4), that

$$
\begin{aligned}
\mathcal{L}_{\delta} v= & -\alpha_{i} \int_{\Gamma}\left(\partial_{\mathbf{n}} u_{\mathrm{int}, 0 \mid \Gamma}+\cdots+\delta^{n} \partial_{\mathbf{n}} u_{\mathrm{int}, n \mid \Gamma}\right) v_{d, 1 \mid \Gamma} d \Gamma \\
& +\alpha_{i} \delta a_{\delta}^{[1]}\left(U_{\mathrm{int}, 0}+\cdots+\delta^{n} U_{\mathrm{int}, n}, v_{d}^{[1]}\right)+\alpha_{i} \int_{\Omega_{\delta, 1}} \nabla R_{1, n} . \nabla v_{d, 1} d \Omega_{\delta, 1} \\
& -\sum_{\beta=1}^{2} \alpha_{\delta} \delta a_{\delta}^{[\beta]}\left(u_{0}^{[\beta]}+\cdots+\delta^{n} u_{n}^{[\beta]}, v_{d}^{[\beta]}\right)+\alpha_{e} \int_{\Omega_{\delta, 2}} \nabla R_{2, n} . \nabla v_{d, 2} d \Omega_{\delta, 2} \\
& +\alpha_{e} \delta a_{\delta}^{[2]}\left(U_{\mathrm{ext}, 0}+\cdots+\delta^{n} U_{\mathrm{ext}, n}, v_{d}^{[2]}\right)-\alpha_{\delta} \int_{\Omega_{\delta}} \nabla \mathcal{P} R . \nabla v_{d} d \Omega_{\delta} \\
& +\alpha_{e} \int_{\Gamma}\left(\partial_{\mathbf{n}} u_{\mathrm{ext}, 0 \mid \Gamma}+\cdots+\delta^{n} \partial_{\mathbf{n}} u_{\mathrm{ext}, n \mid \Gamma}\right) v_{d, 2 \mid \Gamma} d \Gamma .
\end{aligned}
$$

Now, we use the fact that $u_{0}^{[\beta]}, \ldots, u_{n+1}^{[\beta]}(\beta=1,2)$ are solutions of Equations (4.16)-(4.19), and obtain

$$
\begin{aligned}
\mathcal{L}_{\delta} v= & \delta^{n+1}\left\{\delta^{-1} a_{0,2}^{[1]}\left(\alpha_{\delta} u_{n+1}^{[1]}-\alpha_{i} U_{\mathrm{int}, n+1}, v_{d}^{[1]}\right)\right. \\
& -\left(a_{2,2}^{[1]}+a_{0,1}^{[1]}\right)\left(\alpha_{\delta} u_{n}^{[1]}-\alpha_{i} U_{\mathrm{int}, n}, v_{d}^{[1]}\right) \\
& -a_{1,1}^{[1]}\left(\alpha_{\delta} u_{n-1}^{[1]}-\alpha_{i} U_{\mathrm{int}, n-1}+\delta \alpha_{\delta} u_{n}^{[1]}-\delta \alpha_{i} U_{\mathrm{int}, n}, v_{d}^{[1]}\right) \\
& -a_{2,1}^{[1]}\left(\alpha_{\delta} u_{n-2}^{[1]}-\alpha_{i} U_{\mathrm{int}, n-2}+\delta \alpha_{\delta} u_{n-1}^{[1]}-\delta \alpha_{i} U_{\mathrm{int}, n-1}\right. \\
& \left.+\delta^{2} \alpha_{\delta} u_{n}^{[1]}-\delta^{2} \alpha_{i} U_{\mathrm{int}, n}, v_{d}^{[1]}\right)-\cdots-a_{n-1,1}^{[1]}\left(\alpha_{\delta} u_{1}^{[1]}-\alpha_{i} U_{\mathrm{int}, 1}\right. \\
& \left.+\cdots+\delta^{n-1} \alpha_{\delta} u_{n-1}^{[1]}-\delta^{n-1} \alpha_{i} U_{\mathrm{int}, n-1}, v_{d}^{[1]}\right) \\
& -r_{n}^{[1]}\left(\delta ; \alpha_{\delta} u_{1}^{[1]}-\alpha_{i} U_{\mathrm{int}, 1}+\cdots+\delta^{n} \alpha_{\delta} u_{n}^{[1]}-\delta^{n} \alpha_{i} U_{\mathrm{int}, n}, v_{d}^{[1]}\right) \\
& +\delta^{-1} a_{0,2}^{[2]}\left(\alpha_{\delta} u_{n+1}^{[2]}-\alpha_{e} U_{\mathrm{ext}, n+1}, v_{d}^{[2]}\right)
\end{aligned}
$$




$$
\begin{aligned}
& -\left(a_{2,2}^{[2]}+a_{0,1}^{[2]}\right)\left(\alpha_{\delta} u_{n}^{[2]}-\alpha_{e} U_{\text {ext }, n}, v_{d}^{[2]}\right) \\
& -a_{1,1}^{[2]}\left(\alpha_{\delta} u_{n-1}^{[2]}-\alpha_{e} U_{\text {ext }, n-1}+\delta \alpha_{\delta} u_{n}^{[2]}-\delta \alpha_{e} U_{\text {ext }, n}, v_{d}^{[2]}\right) \\
& -a_{2,1}^{[2]}\left(\alpha_{\delta} u_{n-2}^{[2]}-\alpha_{e} U_{\text {ext }, n-2}+\delta \alpha_{\delta} u_{n-1}^{[2]}-\delta \alpha_{e} U_{\text {ext }, n-1}\right. \\
& \left.+\delta^{2} \alpha_{\delta} u_{n}^{[2]}-\delta^{2} \alpha_{e} U_{\text {ext }, n}, v_{d}^{[2]}\right)-\cdots-a_{n-1,1}^{[2]}\left(\alpha_{\delta} u_{1}^{[2]}-\alpha_{e} U_{\text {ext }, 1}\right. \\
& \left.+\cdots+\delta^{n-1} \alpha_{\delta} u_{n-1}^{[2]}-\delta^{n-1} \alpha_{e} U_{\text {ext }, n-1}, v_{d}^{[2]}\right) \\
& \left.-r_{n}^{[2]}\left(\delta ; \alpha_{\delta} u_{1}^{[2]}-\alpha_{e} U_{\text {ext }, 1}+\cdots+\delta^{n} \alpha_{\delta} u_{n}^{[2]}-\delta^{n} \alpha_{e} U_{\text {ext }, n}, v_{d}^{[2]}\right)\right\} \\
& +\alpha_{i} \int_{\Omega_{\delta, 1}} \nabla R_{1, n} . \nabla v_{d, 1} d \Omega_{\delta, 1}+\alpha_{e} \int_{\Omega_{\delta, 2}} \nabla R_{2, n} . \nabla v_{d, 2} d \Omega_{\delta, 2} \\
& -\alpha_{\delta} \int_{\Omega_{\delta}} \nabla \mathcal{P} R . \nabla v_{d} d \Omega_{\delta} .
\end{aligned}
$$

By the estimates based on the explicit expressions of the bilinear form $a_{k, l}^{[\beta]}(.,$. and those of Propositions 1, we have

$$
\begin{aligned}
\left|\mathcal{L}_{\delta} v\right| \leq & c \delta^{n+1} \sum_{\beta=1}^{2}\left(\left\|\nabla_{\Gamma} v_{d}^{[\beta]}\right\|_{L^{2}\left(\Omega^{\beta}\right)}+\delta^{-1}\left\|\partial_{s_{\beta}} v_{d}^{[\beta]}\right\|_{L^{2}\left(\Omega^{\beta}\right)}+\left\|v_{d}^{[\beta]}\right\|_{L^{2}\left(\Omega^{\beta}\right)}\right) \\
& +c \delta^{n}\left\|v_{d}\right\|_{H^{1}\left(\Omega_{\delta, \beta}\right)} .
\end{aligned}
$$

This implies

$$
\begin{aligned}
\left|\mathcal{L}_{\delta} v\right| \leq & c \delta^{n+\frac{1}{2}} \sum_{\beta=1}^{2}\left(\delta^{\frac{1}{2}}\left\|\nabla_{\Gamma} v_{d}^{[\beta]}\right\|_{L^{2}\left(\Omega^{\beta}\right)}+\delta^{-\frac{1}{2}}\left\|\partial_{s_{\beta}} v_{d}^{[\beta]}\right\|_{L^{2}\left(\Omega^{\beta}\right)}\right. \\
& \left.+\delta^{\frac{1}{2}}\left\|v_{d}^{[\beta]}\right\|_{L^{2}\left(\Omega^{\beta}\right)}\right)+c \delta^{n}\left\|v_{d}\right\|_{H^{1}\left(\Omega_{\delta, \beta}\right)} .
\end{aligned}
$$

Then

$$
\left|\mathcal{L}_{\delta} v\right| \leq c \delta^{n}\|v\|_{H^{1}(\Omega)}, \quad \forall v \in H_{0}^{1}(\Omega) .
$$

We set in (5.5) $v_{\mathrm{int}, \delta}=r_{\mathrm{int}, \delta}^{n}, v_{d}=r_{d, \delta}^{n}-\mathcal{P} R$ and $v_{\mathrm{ext}, \delta}=r_{\mathrm{ext}, \delta}^{n}$, we obtain

$$
\left\|r_{\mathrm{int}, \delta}^{n}\right\|_{H^{1}\left(\Omega_{\mathrm{int}, \delta}\right)}+\left\|r_{d, \delta}^{n}-\mathcal{P} R\right\|_{H^{1}\left(\Omega_{\delta}\right)}+\left\|r_{\mathrm{ext}, \delta}^{n}\right\|_{H^{1}\left(\Omega_{\mathrm{ext}, \delta}\right)} \stackrel{(5.6)}{\leq} c \delta^{n} .
$$

Thanks to Proposition 1, we find

$$
\left\|r_{\mathrm{int}, \delta}^{n}\right\|_{H^{1}\left(\Omega_{\mathrm{int}, \delta}\right)}+\left\|r_{d, \delta}^{n}\right\|_{H^{1}\left(\Omega_{\delta}\right)}+\left\|r_{\mathrm{ext}, \delta}^{n}\right\|_{H^{1}\left(\Omega_{\mathrm{ext}, \delta}\right)} \leq c \delta^{n} .
$$

Moreover, since $f$ is $\mathcal{C}^{\infty}$ and for every integer $j$, we have $\left\|u_{\mathrm{ext}, j}\right\|_{H^{1}\left(\Omega_{\mathrm{ext}, \delta}\right)}=$ $O(1),\left\|u_{\mathrm{int}, j}\right\|_{H^{1}\left(\Omega_{\mathrm{int}, \delta}\right)}=O(1)$ and $\left\|u_{d_{\beta}, j}\right\|_{H^{1}\left(\Omega_{\delta, \beta}\right)}=O\left(\delta^{-1 / 2}\right)$, therefore (see, e.g., $[25])$

$$
\begin{aligned}
\left\|r_{\mathrm{int}, \delta}^{n}\right\|_{H^{1}\left(\Omega_{\mathrm{int}, \delta}\right)} & =\left\|\delta^{n+1} u_{\mathrm{int}, n+1}+r_{\mathrm{int}, \delta}^{n+1}\right\|_{H^{1}\left(\Omega_{\mathrm{int}, \delta}\right)} \\
& \stackrel{(5.7)}{\leq} c \delta^{n+1}+c \delta^{n+1} \leq c \delta^{n+1},
\end{aligned}
$$




$$
\begin{aligned}
& \left\|r_{\mathrm{ext}, \delta}^{n}\right\|_{H^{1}\left(\Omega_{\mathrm{ext}, \delta}\right)}=\left\|\delta^{n+1} u_{\mathrm{ext}, n+1}+r_{\mathrm{ext}, \delta}^{n+1}\right\|_{H^{1}\left(\Omega_{\mathrm{ext}, \delta}\right)} \\
& \quad \stackrel{(5.7)}{\leq} c \delta^{n+1}+c \delta^{n+1} \leq c \delta^{n+1}, \\
& \left\|r_{d, \delta}^{n}\right\|_{H^{1}\left(\Omega_{\delta}\right)}=\left\|r_{d, \delta}^{n+1}+\delta^{n+1} u_{d, n+1}\right\|_{H^{1}\left(\Omega_{\delta}\right)} \stackrel{(5.7)}{\leq} c \delta^{n+1}+c \delta^{n+1 / 2} \leq c \delta^{n+1 / 2} .
\end{aligned}
$$

This completes the proof.

\section{Approximate Transmission Conditions}

This section is devoted to the approximation of $u_{\delta}$ by a solution of a problem modelling the effect of the thin layer with a precision of order two in $\delta$. We truncate the series defining the asymptotic expansions, keeping only the first two terms

$$
\begin{aligned}
u_{\mathrm{int}, \delta} & \simeq u_{\mathrm{int}, \delta}^{(1)}=u_{\mathrm{int}, 0}+\delta u_{\mathrm{int}, 1} \text { in } \Omega_{\mathrm{int}}, \\
u_{\mathrm{ext}, \delta} & \simeq u_{\mathrm{ext}, \delta}^{(1)}=u_{\mathrm{ext}, 0}+\delta u_{\mathrm{ext}, 1} \text { in } \Omega_{\mathrm{ext}}, \\
u_{d_{1}, \delta}(x) & \simeq u_{d_{1}, \delta}^{(1)}\left(m, s_{1}\right)=u_{0}^{[1]}\left(m, s_{1}\right)+\delta u_{1}^{[1]}\left(m, s_{1}\right) \quad \forall x=\Phi_{1}\left(m, s_{1}\right) \in \Omega_{\delta, 1}, \\
u_{d_{2}, \delta}(x) & \simeq u_{d_{2}, \delta}^{(1)}\left(m, s_{2}\right):=u_{0}^{[2]}\left(m, s_{2}\right)+\delta u_{1}^{[2]}\left(m, s_{2}\right) \quad \forall x=\Phi_{2}\left(m, s_{2}\right) \in \Omega_{\delta, 2},
\end{aligned}
$$

where

$$
U_{\delta}^{(1)}:=\left\{\begin{array}{l}
u_{\mathrm{ext}, \delta}^{(1)} \text { in } \Omega_{\mathrm{ext}} \\
u_{\mathrm{int}, \delta}^{(1)} \text { in } \Omega_{\mathrm{int}}
\end{array}\right.
$$

is the solution of

$$
\begin{cases}-\operatorname{div}\left(\alpha_{i} \nabla u_{\mathrm{int}, \delta}^{(1)}\right)=f_{\mid \Omega_{\mathrm{int}}} & \text { in } \Omega_{\mathrm{int}}, \\ -\operatorname{div}\left(\alpha_{e} \nabla u_{\mathrm{ext}, \delta}^{(1)}\right)=f_{\mid \Omega_{\mathrm{ext}}} & \text { in } \Omega_{\mathrm{ext}}, \\ u_{\mathrm{int}, \delta \mid \Gamma}^{(1)}-u_{\mathrm{ext}, \delta \mid \Gamma}^{(1)}=\delta \mathcal{A}\left(u_{\mathrm{int}, \delta}^{(1)}\right)-\delta^{2} \xi_{\delta} & \text { on } \Gamma \\ \alpha_{i} \partial_{\mathbf{n}} u_{\mathrm{int}, \delta \mid \Gamma}^{(1)}-\alpha_{e} \partial_{\mathbf{n}} u_{\mathrm{ext}, \delta \mid \Gamma}^{(1)}=\delta \mathcal{B}\left(u_{\mathrm{int}, \delta}^{(1)}\right)-\delta^{2} \rho_{\delta} & \text { on } \Gamma \\ u_{\mathrm{ext}, \delta \mid \partial \Omega}^{(1)}=0 & \text { on } \partial \Omega\end{cases}
$$

with

$$
\begin{aligned}
\mathcal{A}(u) & :=\left[p_{1}\left(1-\alpha_{i} \alpha_{\delta}^{-1}\right)+p_{2}\left(\alpha_{i} \alpha_{e}^{-1}-\alpha_{i} \alpha_{\delta}^{-1}\right)\right]\left(\partial_{\mathbf{n}} u_{\mid \Gamma}\right), \\
\mathcal{B}(u) & :=\left[p_{1}\left(\alpha_{\delta}-\alpha_{i}\right)+p_{2}\left(\alpha_{\delta}-\alpha_{e}\right)\right] \Delta_{\Gamma} u_{\mid \Gamma}, \\
\xi_{\delta} & :=\left[p_{1}\left(1-\alpha_{i} \alpha_{\delta}^{-1}\right)+p_{2}\left(\alpha_{i} \alpha_{e}^{-1}-\alpha_{i} \alpha_{\delta}^{-1}\right)\right] \partial_{\mathbf{n}} u_{\mathrm{int}, 1 \mid \Gamma}, \\
\rho_{\delta} & :=\left[p_{1}\left(\alpha_{\delta}-\alpha_{i}\right)+p_{2}\left(\alpha_{\delta}-\alpha_{e}\right)\right] \Delta_{\Gamma} u_{\mathrm{int}, 1 \mid \Gamma} .
\end{aligned}
$$

Let

$$
U_{\delta}^{a p}:=\left\{\begin{array}{l}
u_{\mathrm{ext}, \delta}^{a p} \text { in } \Omega_{\mathrm{ext}}, \\
u_{\mathrm{int}, \delta}^{a p} \text { in } \Omega_{\mathrm{int}}
\end{array}\right.
$$


be the solution of $(6.1)$ with $\rho_{\delta}=0$ and $\xi_{\delta}=0$. We obtain a problem $\left(\mathcal{P}_{\delta}^{a p}\right)$ with transmission conditions of order equal to that of the differential operator. The new transmission conditions on $\Gamma$ are defined by

$$
\left\{\begin{array}{l}
u_{\mathrm{int}, \delta \mid \Gamma}^{a p}-u_{\mathrm{ext}, \delta \mid \Gamma}^{a p}=\delta\left[p_{1}\left(1-\alpha_{i} \alpha_{\delta}^{-1}\right)+p_{2}\left(\alpha_{i} \alpha_{e}^{-1}-\alpha_{i} \alpha_{\delta}^{-1}\right)\right] \partial_{\mathbf{n}} u_{\mathrm{int}, \delta \mid \Gamma}^{a p} \\
\alpha_{i} \partial_{\mathbf{n}} u_{\mathrm{int}, \delta \mid \Gamma}^{a p}-\alpha_{e} \partial_{\mathbf{n}} u_{\mathrm{ext}, \delta \mid \Gamma}^{a p}=\delta\left[p_{1}\left(\alpha_{\delta}-\alpha_{i}\right)+p_{2}\left(\alpha_{\delta}-\alpha_{e}\right)\right] \Delta_{\Gamma} u_{\mathrm{int}, \delta \mid \Gamma}^{a p}
\end{array}\right.
$$

However, it is not obvious if the bilinear form associated to Problem $\left(\mathcal{P}_{\delta}^{a p}\right)$ is positive or negative. Then the existence and uniqueness of the solution are not ensured by the Lax-Milgram lemma. Therefore, we reformulate Problem $\left(\mathcal{P}_{\delta}^{a p}\right)$ into a nonlocal equation on the interface $\Gamma$ (cf. [5]). A direct use of transmission conditions (6.2) leads to an operator which is not self-adjoint. So, we choose the position of $\Gamma$ in such a way that the jump of the trace of the solution on $\Gamma$ is null. We put

$$
p_{1}\left(1-\alpha_{i} \alpha_{\delta}^{-1}\right)+p_{2}\left(\alpha_{i} \alpha_{e}^{-1}-\alpha_{i} \alpha_{\delta}^{-1}\right)=0
$$

and obtain

$$
p_{1}=\frac{\alpha_{i}\left(\alpha_{e}-\alpha_{\delta}\right)}{\alpha_{\delta}\left(\alpha_{e}-\alpha_{i}\right)} \quad \text { and } \quad p_{2}=\frac{\alpha_{e}\left(\alpha_{\delta}-\alpha_{i}\right)}{\alpha_{\delta}\left(\alpha_{e}-\alpha_{i}\right)},
$$

which is valid only when $\alpha_{i}<\alpha_{\delta}<\alpha_{e}$ or $\alpha_{e}<\alpha_{\delta}<\alpha_{i}$. This corresponds to the case of mid-diffusion. Transmission conditions (6.2) become

$$
\left\{\begin{array}{l}
u_{\mathrm{int}, \delta \mid \Gamma}^{a p}-u_{\mathrm{ext}, \delta \mid \Gamma}^{a p}=0 \\
\alpha_{i} \partial_{\mathbf{n}} u_{\mathrm{int}, \delta \mid \Gamma}^{a p}-\alpha_{e} \partial_{\mathbf{n}} u_{\mathrm{ext}, \delta \mid \Gamma}^{a p}=\delta \frac{\left(\alpha_{e}-\alpha_{\delta}\right)\left(\alpha_{i}-\alpha_{\delta}\right)}{\alpha_{\delta}} \Delta_{\Gamma} u_{\mathrm{int}, \delta}^{a p}
\end{array}\right.
$$

After, we remove the right-hand side of Problem $\left(\mathcal{P}_{\delta}^{a p}\right)$ by a standard lift: let $G$ be in $H_{0}^{1}(\Omega)$ such that $-\operatorname{div}\left(\alpha_{0} \nabla G\right)=f$. Then the function $\Psi=U_{\delta}^{a p}-G$ solves the following problem

$$
\begin{cases}-\operatorname{div}\left(\alpha_{i} \nabla \Psi_{\mathrm{int}}\right)=0 & \text { in } \Omega_{\mathrm{int}} \\ -\operatorname{div}\left(\alpha_{e} \nabla \Psi_{\mathrm{ext}}\right)=0 & \text { in } \Omega_{\mathrm{ext}} \\ \Psi_{\mathrm{int} \mid \Gamma}-\Psi_{\mathrm{ext} \mid \Gamma}=0 & \text { on } \Gamma \\ \alpha_{i} \partial_{\mathbf{n}} \Psi_{\mathrm{int} \mid \Gamma}-\alpha_{e} \partial_{\mathbf{n}} \Psi_{\mathrm{ext} \mid \Gamma}-\delta \frac{\left(\alpha_{e}-\alpha_{\delta}\right)\left(\alpha_{i}-\alpha_{\delta}\right)}{\alpha_{\delta}} \Delta_{\Gamma} \Psi_{\mathrm{int} \mid \Gamma}=g & \text { on } \Gamma \\ \Psi_{\mathrm{ext} \mid \partial \Omega}=0 & \text { on } \partial \Omega\end{cases}
$$

where $g=\left(\alpha_{e}-\alpha_{i}\right) \partial_{\mathbf{n}} G_{\mid \Gamma}+\delta \frac{\left(\alpha_{e}-\alpha_{\delta}\right)\left(\alpha_{i}-\alpha_{\delta}\right)}{\alpha_{\delta}} \Delta_{\Gamma} G_{\mid \Gamma}, \Psi_{\text {int }}:=\Psi_{\mid \Omega_{\text {int }}}$ and $\Psi_{\text {ext }}:=$ $\Psi_{\mid \Omega_{\text {ext }}}$.

We introduce the Steklov-Poicaré operators $S_{\text {int }}$ and $S_{\text {ext }}$ (called also Dirichlet-to-Neumann operators) defined from $H^{1 / 2}(\Gamma)$ onto $H^{-1 / 2}(\Gamma)$ by $S_{\text {int }} \varphi:=$ $\partial_{\mathbf{n}} u_{\mathrm{int} \mid \Gamma}$, where $u_{\text {int }}$ is the solution of the boundary value problem

$$
\begin{cases}-\Delta u_{\text {int }}=0 & \text { in } \Omega_{\text {int }} \\ u_{\text {int } \mid \Gamma=\varphi} & \text { on } \Gamma\end{cases}
$$


and by $S_{\text {ext }} \psi:=\partial_{-\mathbf{n}} u_{\text {ext } \mid \Gamma}$, where $u_{\text {ext }}$ is the solution of the boundary value problem

$$
\begin{cases}-\Delta u_{\mathrm{ext}}=0 & \text { in } \Omega_{\mathrm{ext}} \\ u_{\mathrm{ext} \mid \Gamma}=\psi & \text { on } \Gamma \\ u_{\mathrm{ext} \mid \partial \Omega}=0 & \text { on } \partial \Omega\end{cases}
$$

Then $\left(\mathcal{P}_{\delta}^{a p}\right)$ is equivalent to the boundary equation

$$
\alpha_{i} S_{\mathrm{int}} \omega+\alpha_{e} S_{\mathrm{ext}} \omega-\delta \frac{\left(\alpha_{e}-\alpha_{\delta}\right)\left(\alpha_{i}-\alpha_{\delta}\right)}{\alpha_{\delta}} \Delta_{\Gamma} \omega=g \text { on } \Gamma,
$$

where $\omega$ is the trace of $\Psi$ on the surface $\Gamma$. We are now in position to state the existence and uniqueness theorem, whose proof is similar to that of Theorem 2.5 in $[5]$.

Theorem 5. The operator $P_{\delta}:=\delta \frac{\left(\alpha_{e}-\alpha_{\delta}\right)\left(\alpha_{i}-\alpha_{\delta}\right)}{\alpha_{\delta}} \Delta_{\Gamma}-\alpha_{i} S_{\text {int }}-\alpha_{e} S_{\text {ext }}$ is an elliptic self-adjoint semi-bounded from below pseudodifferential operator of order 2. Moreover, there exists series $\left(\lambda_{n}\right)_{n \in \mathbb{N}}$ growing to infinity such that for any $g \in H^{s}(\Gamma)$ with $s \in \mathbb{R}$, we have the following:

1. If $0 \notin\left(\lambda_{n}\right)_{n \in \mathbb{N}}$, then equation $-P_{\delta} \omega=g$ admits a unique solution in $\mathcal{S}^{\prime}(\Gamma)$ which, in addition, belongs to $H^{s+2}(\Gamma)$;

2. If $0 \in\left(\lambda_{n}\right)_{n \in \mathbb{N}}$, then there is either no solution or a complete affine finite dimensional space of $H^{s+2}(\Gamma)$ solutions.

Finally, we give an error estimate between the solution $u_{\delta}$ of (1.1) and the approximate solution $u_{\delta}^{a p}$ defined on $\Omega$ by

$$
u_{\delta}^{a p}:=\left\{\begin{array}{cl}
u_{\mathrm{int}, \delta}^{a p} & \text { in } \Omega_{\mathrm{int}, \delta}, \\
u_{d, \delta}^{a p} & \text { in } \Omega_{\delta}, \\
u_{\mathrm{ext}, \delta}^{a p} & \text { in } \Omega_{\mathrm{ext}, \delta}
\end{array}\right.
$$

where

$$
\begin{aligned}
u_{d, \delta \mid \Omega_{\delta, 1}}^{a p}(x) & :=u_{d_{1}, \delta}^{a p}(x):=u_{d_{1}, \delta}^{[1], a p}\left(m, s_{1}\right) \\
& :=u_{\mathrm{int}, \delta \mid \Gamma}^{a p}+\delta \frac{\alpha_{i}\left(\alpha_{e}-\alpha_{\delta}\right)}{\alpha_{\delta}\left(\alpha_{e}-\alpha_{i}\right)}\left[\left(s_{1}+1\right) \frac{\alpha_{i}}{\alpha_{\delta}}-1\right] \partial_{\mathbf{n}} u_{\mathrm{int}, \delta \mid \Gamma}^{a p}, \\
u_{d, \delta \mid \Omega_{\delta, 2}}^{a p}(x) & :=u_{d_{2}, \delta}^{a p}(x):=u_{d_{2}, \delta}^{[2], a p}\left(m, s_{2}\right) \\
& :=u_{\mathrm{ext}, \delta \mid \Gamma}^{a p}+\delta \frac{\alpha_{e}\left(\alpha_{\delta}-\alpha_{i}\right)}{\alpha_{\delta}\left(\alpha_{e}-\alpha_{i}\right)}\left[\left(s_{2}-1\right) \frac{\alpha_{e}}{\alpha_{\delta}}+1\right] \partial_{\mathbf{n}} u_{\mathrm{ext}, \delta \mid \Gamma}^{a p} .
\end{aligned}
$$

We can now formulate our main result.

Theorem 6. There exists a constant c independent of $\delta$ such as

$$
\begin{aligned}
& \left\|u_{i n t, \delta}-u_{i n t, \delta}^{a p}\right\|_{H^{1}\left(\Omega_{i n t, \delta}\right)}+\delta^{\frac{1}{2}}\left\|u_{d, \delta}-u_{d, \delta}^{a p}\right\|_{H^{1}\left(\Omega_{\delta}\right)} \\
& \quad+\left\|u_{e x t, \delta}-u_{e x t, \delta}^{a p}\right\|_{H^{1}\left(\Omega_{e x t, \delta}\right)} \leq c \delta^{2} .
\end{aligned}
$$


Proof. Since

$$
\begin{aligned}
& \left\|u_{d_{1}, \delta}^{[1], a p}-u_{d_{1}, \delta}^{(1)}\right\|_{H^{1}\left(\Omega^{1}\right)} \leq c\left(\left\|u_{\mathrm{int}, \delta}^{a p}-u_{\mathrm{int}, \delta}^{(1)}\right\|_{H^{1}\left(\Omega_{\mathrm{int}}\right)}+\delta^{2}\left\|u_{\mathrm{int}, 1}\right\|_{H^{1}\left(\Omega_{\mathrm{int}}\right)}\right), \\
& \left\|u_{d_{2}, \delta}^{[2], a p}-u_{d_{2}, \delta}^{(1)}\right\|_{H^{1}\left(\Omega^{2}\right)} \leq c\left(\left\|u_{\mathrm{ext}, \delta}^{a p}-u_{\mathrm{ext}, \delta}^{(1)}\right\|_{H^{1}\left(\Omega_{\mathrm{ext}}\right)}+\delta^{2}\left\|u_{\mathrm{ext}, 1}\right\|_{H^{1}\left(\Omega_{\mathrm{ext}}\right)}\right),
\end{aligned}
$$

where $c$ is a constant independent of $\delta$ and according to the Convergence Theorem, it is sufficient to estimate the error $U_{\delta}^{a p}-U_{\delta}^{(1)}$. Therefore, as in [28, p. 36], we perform an asymptotic expansion for $U_{\delta}^{a p}$. The ansatz

$$
U_{\delta}^{a p}=\sum_{j \geq 0} \delta^{j} w_{j},
$$

where $w_{j \mid \Omega_{\mathrm{ext}}}:=w_{\mathrm{ext}, j}$ and $w_{j \mid \Omega_{\mathrm{int}}}:=w_{\mathrm{int}, j}$, gives the recurrence relations

$$
\begin{cases}-\operatorname{div}\left(\alpha_{i} \nabla w_{\mathrm{int}, j}\right)=\delta_{j, 0} f_{\mid \Omega_{\mathrm{int}}} & \text { in } \Omega_{\mathrm{int}}, \\ -\operatorname{div}\left(\alpha_{e} \nabla w_{\mathrm{ext}, j}\right)=\delta_{j, 0} f_{\mid \Omega_{\mathrm{ext}}} & \text { in } \Omega_{\mathrm{ext}}, \\ w_{\mathrm{int}, j \mid \Gamma}-w_{\mathrm{ext}, j \mid \Gamma}=0 & \text { on } \Gamma, \\ \alpha_{i} \partial_{\mathbf{n}} w_{\mathrm{int}, j \mid \Gamma}-\alpha_{e} \partial_{\mathbf{n}} w_{\mathrm{ext}, j \mid \Gamma}=\frac{\left(\alpha_{e}-\alpha_{\delta}\right)\left(\alpha_{i}-\alpha_{\delta}\right)}{\alpha_{\delta}} \Delta_{\Gamma} w_{\mathrm{int}, j-1 \mid \Gamma} & \text { on } \Gamma, \\ w_{\mathrm{ext}, j \mid \partial \Omega}=0 & \text { on } \partial \Omega\end{cases}
$$

with the convention that $w_{-1}=0$. A simple calculation shows that the first two terms $w_{0}$ and $w_{1}$ coincide with the first two terms $u_{0}$ and $u_{1}$ of (4.3) and (4.4). Furthermore, since $f$ is $\mathcal{C}^{\infty}$, each term of $(6.3)$ is bounded in $H^{1}(\Omega)$. Then, by setting $\mathcal{R}_{w}:=U_{\delta}^{a p}-w_{0}-\delta w_{1}-\delta^{2} w_{2}$, there exists $c>0$, such as $\left\|\mathcal{R}_{w}\right\|_{H^{1}(\Omega)} \leq c \delta^{2}$, which gives the desired result.

\section{Conclusion}

We derived and justified an asymptotic expansion of the exact solution of Problem (1.1). We successfully modeled the effect of the thin layer by a problem with transmission condition with accuracy up to $O\left(\delta^{2}\right)$.

An interesting perspective is to investigate the cases of high-diffusion i.e. $\alpha_{e}<\alpha_{i}<\alpha_{\delta}$ or $\alpha_{i}<\alpha_{e}<\alpha_{\delta}$, low-diffusion i.e. $\alpha_{\delta}<\alpha_{i}<\alpha_{e}$ or $\alpha_{\delta}<\alpha_{e}<$ $\alpha_{i}$, and the case where diffusion constants depend on $\delta$. Lastly, the cases of Helmholtz and Maxwell equations would be interesting.

\section{Acknowledgment}

The author would like to thank the referees for their valuable remarks and suggestions which led to an improvement of the paper.

\section{References}

[1] E. Acerbi and G. Buttazzo. Reinforcement problems in the calculus of variations. Ann. Inst. H. Poincar Anal. Non Linaire, 3(4):273-284, 1986. 
[2] H. Ammari and J.C. Nédélec. Sur les conditions d'impédance généralisées pour les couches minces. C. R. Acad. Sci. Paris Sér. I Math., 322(10):995-1000, 1996.

[3] A. Bendali and K. Lemrabet. The effect of a thin coating on the scattering of a time-harmonic wave for the Helmholtz equation. SIAM J. Appl. Math., 56(6):1664-1693, 1996. http://dx.doi.org/10.1137/S0036139995281822.

[4] A. Bendali and K. Lemrabet. Asymptotic analysis of the scattering of a timeharmonic electromagnetic wave by a perfectly conducting metal coated with a thin dielectric shell. Asymptot. Anal., 57(3-4):199-227, 2008.

[5] V. Bonnaillie-Noël, M. Dambrine, F. Hérau and G. Vial. On generalized ventcel's type boundary conditions for laplace operator in a bounded domain. SIAM J. Math. Anal., 42(2):931-945, 2010. http://dx.doi.org/10.1137/090756521.

[6] K.E. Boutarene. Asymptotic analysis for a diffusion problem. C. R. Math. Acad. Sci. Paris, 349(1-2):57-60, 2011. http://dx.doi.org/10.1016/j.crma.2010.12.002.

[7] M. Boutkrida, J. Mossino and G. Moussa. On the torsional rigidity problem with nonhomogeneous reinforcement. Papers in memory of Ennio De Giorgi. Ricerche Mat., 48(suppl.):1-24, 1999.

[8] H. Brezis. Functional analysis, Sobolev spaces and partial differential equations. Universitext. Springer, New York, 2011.

[9] A. Brillard and M. El Jarroudi. On the interface boundary conditions between two interacting incompressible viscous fluid flows. J. Differential Equations, 255(5):881-904, 2013. http://dx.doi.org/10.1016/j.jde.2013.04.038.

[10] G. Buttazzo and R. V. Kohn. Reinforcement by a thin layer with oscillating thickness. Appl. Math. Optim., 16(1):247-261, 1987.

http://dx.doi.org/10.1007/BF01442194.

[11] G. Caloz, M. Costabel, M. Dauge and G. Vial. Asymptotic expansion of the solution of an interface problem in a polygonal domain with thin layer. Asymptot. Anal., 50(1-2):121-173, 2006.

[12] J. Casado-Díaz, M. Luna-Laynez and F.J. Suárez-Grau. A viscous fluid in a thin domain satisfying the slip condition on a slightly rough boundary. C. R. Math., 348(17-18):967-971, 2010. http://dx.doi.org/10.1016/j.crma.2010.07.023.

[13] B. Delourme, H. Haddar and P. Joly. Approximate models for wave propagation across thin periodic interfaces. J. Math. Pures Appl. (9), 98(1):28-71, 2012.

[14] M.P. Do Carmo. Differential Geometry of Curves and Surfaces. Prentice-Hall Inc., Englewood Cliffs, NJ, 1976.

[15] M. Duruflé, H. Haddar and P. Joly. High order generalized impedance boundary conditions in electromagnetic scattering problems. C.R. Phys., 7(5):533-542, 2006. http://dx.doi.org/10.1016/j.crhy.2006.03.010.

[16] B. Engquist and J.C. Nédélec. Effective boundary conditions for acoustic and electromagnetic scattering in thin layers. Research Report CMAP 278, Ecole Polytechnique, France, 1993.

[17] A. Gaudiello and K. Hamdache. The polarization in a ferroelectric thin film: local and nonlocal limit problems. ESAIM Control Optim. Calc. Var., 19:657667, 2013. http://dx.doi.org/10.1051/cocv/2012026. 
[18] A. Gaudiello and A. Sili. Asymptotic analysis of the eigenvalues of an elliptic problem in an anisotropic thin multidomain. Proc. Roy. Soc. Edinburgh Sect. A Math., 141:739-754, 2011.

[19] J.C. Nédélec. Acoustic and Electromagnetic Equations: Integral Representations for Harmonic Problems, volume 144. Springer-Verlag, 2001.

[20] C. Poignard. Méthodes asymptotiques pour le calcul des champs électromagnétiques dans des milieux à couches minces. Application aux cellules biologiques. PhD thesis, Université Claude Bernard-Lyon 1, 2006.

[21] C. Poignard. About the transmembrane voltage potential of a biological cell in time-harmonic regime. In Mathematical Methods for Imaging and Inverse Problems, volume 26 of ESAIM Proc., pp. 162-179. EDP Sci., Les Ulis, 2009.

[22] C. Poignard. Approximate transmission conditions through a weakly oscillating thin layer. Math. Methods Appl. Sci., 32(4):435-453, 2009. http://dx.doi.org/10.1002/mma.1045.

[23] C. Poignard. Boundary layer correctors and generalized polarization tensor for periodic rough thin layers. A review for the conductivity problem. ESAIM Proc., 37:136-165, 2012.

[24] K. Schmidt. High-order numerical modeling of highly conductive thin sheets. PhD thesis, ETH Zurich, 2008.

[25] K. Schmidt and S. Tordeux. Asymptotic modelling of conductive thin sheets. $Z$. Angew. Math. Phys., 61(4):603-626, 2010.

http://dx.doi.org/10.1007/s00033-009-0043-x.

[26] K. Schmidt and S. Tordeux. High order transmission conditions for thin conductive sheets in magneto-quasistatics. ESAIM Math. Model. Numer. Anal., 45(6):1115-1140, 2011. http://dx.doi.org/10.1051/m2an/2011009.

[27] J. Shewchuk. What is a good linear finite element? interpolation, conditioning, anisotropy, and quality measures. Preprint, University of California at Berkeley, 2002 .

[28] G. Vial. Analyse multi-échelle et conditions aux limites approchées pour un problème avec couche mince dans un domaine à coin. $\mathrm{PhD}$ thesis, Université de Renne 1, France, 2003. 